\title{
The First Spectrum of Barium, Ba I
}

\author{
Henry Norris Russell ${ }^{1}$ and Charlotte E. Moore
}

\begin{abstract}
The analysis of the $\mathrm{Ba}$ I spectrum is presented. Although a monograph based on homogeneous observations is needed, this paper has been prepared from existing references, to present unpublished analysis by the senior author and, also, because Ba I is important in the history of atomic spectra. The first regularities attributed to two excited electrons were found in $\mathrm{Ba} \mathrm{I}$ as well as in Ca I and $\mathrm{Sr} \mathrm{I}$.

Three tables are included: A complete term list; a complete line list containing all observed and all classified lines; a table giving the estimated intensities of the observed combinations. There are approximately 390 classified lines.

The limit, derived from well-established series, is $42032.4 \mathrm{~K}$, giving an ionization potential of 5.210 volts
\end{abstract}

The Ba I spectrum occupies an important position in the history of atomic spectra. Although regular series terms were reported by Saunders, PaschenGötze, and Fowler (1920 to 1922) [1] the first regularities attributed to two excited electrons were found in Ba I, as well as in Ca I and Sr I, in 1925 [2].

Shenstone and Russell suggested some revisions in the early analysis and discussed the perturbed series of Ba I in 1932 [3]. They adopted as the ionization limit, i. e., the absolute value of the ground state, the value $42032.4 \mathrm{~K}$, giving an ionization potential 5.210 volts

Although the spectrum has been studied thorougbly, a monograph based on a complete homogeneous line list is needed. In view of the fact that this does not appear to be forthcoming, a complete line list and multiplet array have been prepared from the existing literature references, and from unpublished analysis by the senior author. Uniform notation has been adopted, and the decimals of all energy levels have been revised. Sullivan and Burns (table 2, ref. C) improved the values of a number of energy levels from their interferometric measurements of selected lines. Starting with these three-place values, the rest were revised from a study of all observed combinations.

The complete term list is given in table 1 , which is self-explanatory. The terms are listed in the following order:

(1) Terms due to the equivalent electron pairs $6 s^{2}, 6 p^{2}$, and $5 d^{2}$ so far as known.

(2) The regular series of triplet and singlet terms having as limit the ground term in Ba II $6 s{ }^{2} \mathrm{~S}_{01 / 2}$, given in the order of the running electrons $n s, n p$, $n d$, and $n f$.

(3) The triplet and singlet terms produced by two excited electrons, having as limit the term $5 d^{2} \mathrm{D}$ in Ba II, arranged in the same order of running electrons. The designations for these terms have primes attached to the running electrons to denote the higher limit term in $\mathrm{Ba}$ II.

\footnotetext{
Princeton University Observatory, Princeton, N.J.

2 Figures in brackets indicate the literature references at the end of this text.
}

B. E. Moore observed the Zeeman effect in Ba I in 1908 [4] and, so far as is known to the present authors, his work has never been superseded. His measurements of $\Delta \lambda / \lambda^{2}$ have been divided by the correction factor 1.09 , a factor adopted to reduce them to the theoretical Landé patterns for a very few well-known lines. For unresolved patterns the formulas for blends given by Shenstone and Blair [5] have been used to determine observed $g$-values. The results obtained from Moore's observations are as follows:

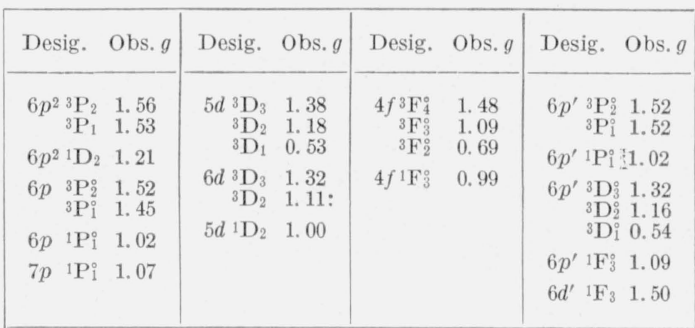

Table 2 contains all of the observed and classified lines of BaI. The letter in column 1 indicates the literature reference from which the wavelength in column 2 is quoted. The bibliography of these references is given at the end of this table. Two sets of intensities are quoted in column 3. The left-hand entry is from the reference source indicated in column 1, except for those in parentheses, which are arc intensities estimated by Exner and Haschek (ref. G). The intensities in parentheses are entered for the lines observed with the interferometer by Burns and Sullivan. The right-hand entries in column 3 are the are intensity and temperature class estimated by A. S. King [6]. Brackets denote King's estimated intensity in the furnace spectrum, in cases where this spectrum gives better resolution than his arc spectrum. Column 4 contains the vacuum wave number in $\mathrm{cm}^{-1}$, labeled $\mathrm{K}$ for kayser. It is followed by $\Delta K$, the difference between the observed wave number and the wave number calculated from the energy levels. The last column gives the multiplet designation in the notation of table 1 . There are approximately 390 classified lines in table 2 . 
TABle 1. Ba I terms

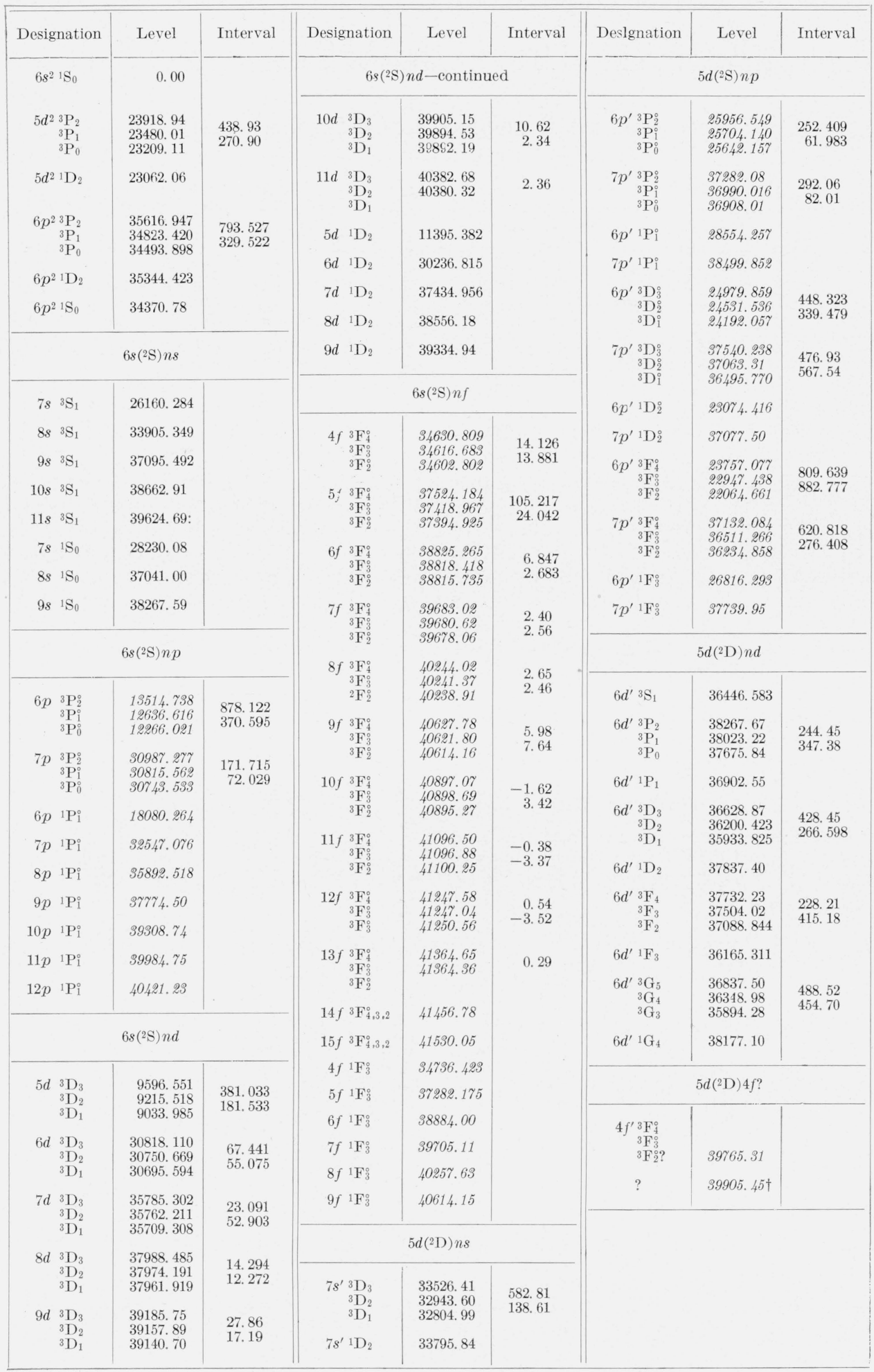

$\dagger J=1$ or 2. 
The intensities of the observed combinations in Ba $\mathrm{I}$ are recorded in table 3 . This table is arranged in two sections: (1) the multiplets produced by transitions from the low even terms $6 s^{2}{ }^{1} \mathrm{~S}, 5 d^{2}{ }^{3} \mathrm{P}, 5 d^{2}{ }^{1} \mathrm{D}$; $5 d^{3} \mathrm{D}$, and $5 d^{1} \mathrm{D}$ (more precisely $6 s 5 d^{3,1} \mathrm{D}$ ), to higher odd terms, the latter being given in the same order as in table 1 ; (2) multiplets representing transitions from $6 p^{3,1} \mathrm{P}^{\circ}$ and $6 p^{\prime}{ }^{3,1} \mathrm{P}^{\circ}, 6 p^{\prime 3,1} \mathrm{D}^{\circ}$, $6 p^{\prime 3,1} \mathrm{~F}^{\circ}$ to higher even terms as given in table 1 . All observed combinations are reported in table 3 except the following four, which are omitted to save space:

\begin{tabular}{|c|c|}
\hline Desig. & Int. \\
\hline 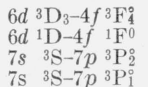 & $\begin{array}{l}(20) \\
(20) \\
(40) \\
(15)\end{array}$ \\
\hline
\end{tabular}

The intensities in table 3 are from column 3 of table 2. King's estimated arc intensity (or furnace intensity in brackets) and temperature class are entered when known. All intensities not by King are given in parentheses. For some lines no estimated intensity is available. In such cases, a "(-)" has been adopted to denote that the combination has been observed.

The present summary of our knowledge of the Ba I spectrum does not serve to replace the monograph needed for this spectrum. Further observations of the Zeeman effect are desirable, With modern infrared detectors the long-wave region beyond the photographic range offers an attractive and worthwhile program. In particular, the even term $5 d^{2}{ }^{3} \mathrm{~F}$ remains to be found. The short-wave region likewise needs to be reobserved, and many of the early measurements throughout the spectrum could be improved.

The authors are particularly indebted to W. F. Meggers for the use of his precise observational data in the photographic long-wave region, in advance of publication.

\section{References}

[1] F. A. Saunders, Astrophys. J. 51, 23 (1920); F. Paschen und R Götze, Seriengesetze der Linienspektren, p. 90, (Julius Springer, Berlin, 1922); A. Fowler, Report on Series in Line Spectra, p. 133 (Fleetway Press, London, 1922).

[2] H. N. Russell and F. A. Saunders, Astrophys. J. 61, 38 (1925).

[3] A. G. Shenstone and H. N. Russell, Phys. Rev. 39, 415 (1932).

[4] B. E. Moore, Ann. der Phys. [4] 25, 309 (1908).

[5] A. G. Shenstone and H. A. Blair, Phil. Mag. 8, 765 (1929); H. N. Russell, Phys. Rev. 36, 1590 (1930).

[6] A. S. King, Contr. Mt. Wilson Solar Obs. No. 150, Astrophys. J. 48, 13 (1918); Contr. Mt. Wilson Obs. No. 181; Astrophys. J. 61, 179 (1920).

Table 2. Observed and classified Ba I lines

Symbols in Intensity column

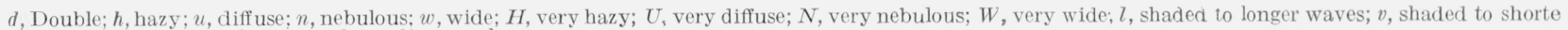
$d$, Double; $h$, hazy; $u$, diff use; $n$, nebulous; $w$, wide;
waves; $r$, partial self-reversal; $R$, complete self-reversal.

\begin{tabular}{|c|c|c|c|c|c|c|c|c|c|c|c|c|c|}
\hline \multirow{2}{*}{$\begin{array}{l}\text { Lit. } \\
\text { ref }\end{array}$} & \multirow{2}{*}{ I. A. } & \multicolumn{2}{|c|}{ Intensity } & \multirow{2}{*}{ K } & \multirow{2}{*}{$\frac{\Delta K}{0-c}$} & \multirow{2}{*}{ Desig. } & \multirow{2}{*}{$\begin{array}{l}\text { lit. } \\
\text { ref. }\end{array}$} & \multirow{2}{*}{ I. A. } & \multicolumn{2}{|c|}{ Intensity } & \multirow{2}{*}{$\mathrm{K}$} & \multirow{2}{*}{$\begin{array}{l}\Delta \mathrm{K} \\
\mathrm{o}-\mathrm{c}\end{array}$} & \multirow{2}{*}{ Desig. } \\
\hline & & Ref. & $\begin{array}{c}\text { King } \\
\text { Are T. C. }\end{array}$ & & & & & & Ref. & $\begin{array}{c}\text { King } \\
\text { Are T. C. }\end{array}$ & & & \\
\hline A & 30933.8 & 30 & -... & 3232 & 0 & $5 d \quad{ }^{3} \mathrm{D}_{1}-6 p{ }^{3} \mathrm{P}_{0}^{\circ}$ & & I. A. & & & & & \\
\hline $\begin{array}{l}\mathrm{A} \\
\mathrm{A}\end{array}$ & $\begin{array}{l}30686.9 \\
30468.5\end{array}$ & $\begin{array}{l}20 \\
15\end{array}$ & & $\begin{array}{l}3258 \\
3281\end{array}$ & -..... & - & B & 11884.51 & $5 h$ & & 8412.01 & -0.05 & $6 p^{\prime}{ }^{3} \mathrm{D}_{2}^{\circ}-7 s^{\prime}{ }^{3} \mathrm{D}_{2}$ \\
\hline $\mathrm{A}$ & 29790.6 & 35 & 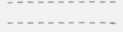 & 3356 & & & B & 11697.45 & $40 h$ & & 8546.53 & -0.02 & \\
\hline $\mathrm{A}$ & 29223.9 & 50 & & 3421 & 0 & $5 d{ }^{3} \mathrm{D}_{2}-6 p{ }^{3} \mathrm{P}_{1}$ & B & 11607.36 & $30 h$ & & 8612.86 & -0.07 & $6 p^{\prime} 3 \mathrm{D}_{\mathrm{i}}^{\circ}-7 s^{\prime}{ }^{3} \mathrm{D}_{1}$ \\
\hline A & 27751. 1 & 30 & .............. & $\begin{array}{l}3602 \\
3813\end{array}$ & $\begin{array}{r}-1 \\
0\end{array}$ & & ${ }_{B}^{B}$ & 11583.0 & $1 h ?$ & & 8631.0 & +0.1 & $6 p^{\prime}{ }^{3} \mathrm{~F}_{2}-6 d{ }^{3} \mathrm{D}_{1}$ \\
\hline $\begin{array}{l}\mathrm{A} \\
\mathrm{A}\end{array}$ & $\begin{array}{l}26221.4 \\
25515.7\end{array}$ & $\begin{array}{l}20 \\
50\end{array}$ & $-\cdots$ & $\begin{array}{l}3813 \\
3918\end{array}$ & $\begin{array}{l}0 \\
0\end{array}$ & $\begin{array}{lll}6 d & { }^{3} \mathrm{D}_{3}-4 f & { }^{3} \mathrm{~F}_{4}^{0} \\
5 d & 3{ }^{3} \mathrm{D}_{3}-6 p & { }^{3} \mathrm{P}_{2}^{0}\end{array}$ & $\begin{array}{l}\mathrm{B} \\
\mathrm{B}\end{array}$ & $\begin{array}{l}11423.46 \\
11403.92\end{array}$ & $\begin{array}{c}20 h \\
2\end{array}$ & & $\begin{array}{l}8751.52 \\
8766.51\end{array}$ & -0.02 & $6 p^{\prime}{ }^{3} \mathrm{D}_{1}^{1}-7 s^{\prime}{ }^{3} \mathrm{D}_{2}$ \\
\hline A & 23255.3 & 30 & & 4299 & 0 & $5 d \quad{ }^{3} \mathrm{D}_{2}-6 p{ }^{3} \mathrm{P}_{2}^{0}$ & B & 11373.88 & $5 h$ & & 8789.67 & -0.09 & $6 p^{\prime}{ }^{3} \mathrm{P}_{1}^{\circ}-6 p^{2}{ }^{3} \mathrm{P}_{0}$ \\
\hline A & 22313.4 & 20 & & 4480 & -1 & $5 d \quad{ }^{3} \mathrm{D}_{1}-6 p{ }^{3} \mathrm{P}_{2}^{0}$ & B & 11303. 04 & 80 & & 8844.75 & 0.00 & $5 d{ }^{3} \mathrm{D}_{2}-6 p \quad{ }^{1} \mathrm{P}_{1}^{\circ}$ \\
\hline A & 22220.8 & 20 & $\ldots$ & 4499 & -1 & $6 d{ }^{1} \mathrm{D}_{2}-4 f{ }^{1} \mathrm{~F}_{3}^{\circ}$ & B & 11274.77 & 10 & & 8866.93 & +0.06 & $6 p^{\prime}{ }^{3} \mathrm{P}_{2}^{0}-6 p^{2}{ }^{3} \mathrm{P}_{1}$ \\
\hline A & & 15 & -... & 4655 & 0 & & ${ }_{B}^{B}$ & 11257.3 & $2 H ?$ & -... & $\begin{array}{l}8880.7 \\
8991.7\end{array}$ & 0.0 & $6 p^{\prime}{ }^{1} \mathrm{P}_{1}^{\circ}-7 d{ }^{1} \mathrm{D}_{2}$ \\
\hline $\begin{array}{l}\mathrm{A} \\
\mathrm{A}\end{array}$ & $\begin{array}{l}20712.0 \\
19987.9\end{array}$ & $\begin{array}{l}40 \\
25\end{array}$ & -.. & $\begin{array}{l}4827 \\
5002\end{array}$ & $\begin{array}{l}0 \\
0\end{array}$ & $\begin{array}{ll}7 s & { }^{3} \mathrm{~S}_{1}-7 p \\
6 p & { }^{1} \mathrm{P}_{1}-5 \mathrm{P}_{2}^{\circ} \\
\mathrm{P}^{2} & { }^{1} \mathrm{D}_{2}\end{array}$ & $\begin{array}{l}B \\
B\end{array}$ & 11114. 42 & $50 h$ & & 8994. 85 & -0.02 & $6 p^{\prime}{ }^{3} \mathrm{D}_{2}^{\circ}-7 s^{\prime}{ }^{3} \mathrm{D}_{3}$ \\
\hline $\mathrm{A}$ & 19074.6 & 20 & & 5241 & -1 & $6 p^{\prime}{ }^{1} \mathrm{P}_{1}^{0}-7 s^{\prime}{ }^{1} \mathrm{D}_{2}$ & B & 11075.7 & 3 & & 9026.3 & 0.0 & $5 d \quad{ }^{3} \mathrm{D}_{1}-6 p \quad{ }^{1} \mathrm{P}_{1}^{0}$ \\
\hline A & 18204.1 & 15 & & 5492 & 0 & $5 d^{2}{ }^{1} \mathrm{D}_{2}-6 p^{\prime}{ }^{1} \mathrm{P}_{1}^{0}$ & B & 11012. 69 & $60 h l$ & & 9077.95 & -0.04 & $6 p^{\prime}{ }^{1} \mathrm{~F}_{3}^{0}-6 d^{\prime}{ }^{3} \mathrm{G}_{3}$ \\
\hline A & $17182.5 ?$ & 5 & & 5818 & +1 & $6 p^{\prime}{ }^{1} \mathrm{P}_{1}-6 p^{2}{ }^{1} \mathrm{~S}_{0}$ & B & 10962.65 & 20 & & 9119.39 & +0.11 & $6 p^{\prime}{ }^{3} \mathrm{P}_{1}^{\circ}-6 p^{2}{ }^{3} \mathrm{P}_{1}$ \\
\hline A & 17064.8 & 10 & (n..... & 5858 & -1 & $6 p{ }^{1} \mathrm{P}_{1}^{0}-5 d^{2}{ }^{3} \mathrm{P}_{2}$ & B & 10914.85 & 3 & & 9159.32 & & 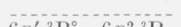 \\
\hline A & 15000.4 & 40 & & $\begin{array}{l}6665 \\
6079\end{array}$ & $\begin{array}{r}0 \\
-1\end{array}$ & $\begin{array}{lll}5 d & { }^{1} \mathrm{D}_{2}-6 p & { }^{1} \mathrm{P}_{1}^{\circ} \\
6 p^{\prime} & 1 \mathrm{~F}_{2}^{\circ}-7 s^{\prime} & 1 \mathrm{D}_{2}\end{array}$ & $\begin{array}{l}\mathrm{B} \\
\mathrm{B}\end{array}$ & $\begin{array}{l}10888.65 \\
10791.25\end{array}$ & $\begin{array}{l}30 \\
40 h l\end{array}$ & -... & $\begin{array}{l}9181.36 \\
9264.23\end{array}$ & $\begin{array}{l}+0.10 \\
-0.07\end{array}$ & $\begin{array}{l}6 p^{\prime}{ }^{3} \mathrm{P}_{0}^{\circ}-6 p^{2}{ }^{3} \mathrm{P}_{1} \\
6 p^{\prime}{ }^{3} \mathrm{D}_{2}^{\circ}-7 s^{\prime} \\
{ }_{1} \mathrm{D}_{2}\end{array}$ \\
\hline A & 14325. 4 & 25 & & 6979 & $\begin{array}{l}-1 \\
-1\end{array}$ & 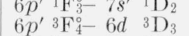 & $\mathrm{B}$ & 10769. 2 & $4 h$ & & $\begin{array}{l}9204.25 \\
9283.2\end{array}$ & $\begin{array}{l}-0.01 \\
+0.1\end{array}$ & $6 p^{\prime}{ }^{1} \mathrm{P}_{1}^{0}-6 d^{\prime}{ }^{1} \mathrm{D}_{2}$ \\
\hline A & 14159.5 & 30 & & 7060 & -8 & $\left(5 \mathrm{~d}^{2}{ }^{3} \mathrm{P}_{2}-7 p{ }^{3} \mathrm{P}_{2}^{\circ}\right)$ & B & 10693. 7 & $8 h l$ & & 9348.8 & -0.2 & $6 p^{\prime}{ }^{1} \mathrm{~F}_{3}^{\circ}-6 d^{\prime}{ }^{1} \mathbf{F}_{3}$ \\
\hline A & 14077.9 & 40 & ............ & 7101 & 0 & $6 p^{\prime}{ }^{3} \mathrm{P}_{1}^{\circ}-7 s^{\prime}{ }^{3} \mathrm{D}_{1}$ & B & 10649.10 & $30 h$ & $\ldots$ & 9387.90 & +0.03 & $6 p^{\prime}{ }^{3} \mathrm{P}_{2}^{0}-6 p^{2}-\mathrm{D}_{2}$ \\
\hline A & 13956.5 & 20 & (n) & $\begin{array}{l}7163 \\
7039\end{array}$ & $\begin{array}{r}0 \\
-1\end{array}$ & $\begin{array}{l}6 p^{\prime}{ }^{3} \mathrm{P}_{0}^{0}-7 s^{\prime}{ }^{3} \mathrm{D}_{1} \\
6 n^{\prime}{ }^{3} \mathrm{P}^{\circ}-7 s^{\prime}\end{array}$ & $\begin{array}{l}\mathrm{B} \\
\mathrm{B}\end{array}$ & $\begin{array}{l}10540.10 \\
10487.3\end{array}$ & $\begin{array}{l}8 \\
2 h\end{array}$ & n & $\begin{array}{l}9484.97 \\
9532.7\end{array}$ & $\begin{array}{c}-0.05 \\
0.0\end{array}$ & $\begin{array}{l}5 d^{2}-\mathrm{D}_{2}-7 p \\
6 p^{\prime}+\mathrm{F}_{3}^{0}-6 d^{\prime} \\
{ }^{3} \mathrm{~F}_{1}\end{array}$ \\
\hline $\begin{array}{l}A \\
A\end{array}$ & $\begin{array}{l}13810.5 \\
13207.3\end{array}$ & $\begin{array}{l}40 \\
40\end{array}$ & (n) & 7569 & -1 & $6 p^{\prime}{ }^{3} \mathrm{P}_{2}^{\circ}-7 s^{\prime}{ }^{3} \mathrm{D}_{3}$ & $\mathrm{~B}$ & 10471. 26 & 100 & & 9547. 34 & $\begin{array}{r}0.0 \\
+0.02\end{array}$ & $\begin{array}{llll}0 p & 3 \mathrm{P}_{2}^{0}-5 d^{2} & 1 & \mathrm{D}_{2}\end{array}$ \\
\hline A & 12814.8 & 10 & & 7801 & -2 & $6 p^{\prime}{ }^{3} \mathrm{~F}_{3}^{0}-6 d{ }^{3} \mathrm{D}_{2}$ & B & 10409.7 & $3 h$ & & 9603.8 & 0.0 & $6 p^{\prime}{ }^{3} \mathrm{D}_{1}-7 s^{\prime}{ }^{1} \mathrm{D}_{2}$ \\
\hline A & 12554.3 & 30 & & 7963 & -1 & $6 p^{\prime} 3 \mathrm{D}_{3}^{\circ}-7 s^{\prime}{ }^{3} \mathrm{D}_{2}$ & B & 10370.35 & $10 h$ & & 9640.23 & -0.05 & $6 p^{\prime}{ }^{3} \mathrm{P}_{1}^{\circ}-6 p^{2}{ }^{1} \mathrm{D}_{2}$ \\
\hline B & 12083. 60 & $1 h$ & & 8273.42 & -0.03 & $6 p^{\prime}{ }^{3} \mathrm{D}_{2}^{\circ}-7 s^{\prime}{ }^{3} \mathrm{D}_{1}$ & B & 10349.05 & $8 h l$ & & 9660.08 & -0.32 & $6 p^{\prime}{ }^{3} \mathrm{P}_{2}^{\circ}-6 p^{2}{ }^{3} \mathrm{P}_{2}$ \\
\hline A & 11978.2 & 15 & & 8346 & -2 & $6 p^{\prime}{ }^{1} \mathrm{P}_{1}^{\circ}-6 d^{\prime}{ }^{1} \mathrm{P}_{1}$ & B & 10327.4 & & & 9680.3 & ...... & - not \\
\hline
\end{tabular}


Table 2-Continued

\begin{tabular}{|c|c|c|c|c|c|c|c|c|c|c|c|c|c|}
\hline \multirow{2}{*}{$\begin{array}{l}\text { Lit. } \\
\text { Ref. }\end{array}$} & \multirow{2}{*}{ I. A. } & In & ensity & & & & & & & tensity & & & \\
\hline & & Ref. & $\begin{array}{l}\text { King } \\
\text { Are T. C. }\end{array}$ & $\mathrm{K}$ & $0-\mathrm{c}$ & Desig. & Ref. & 1. A. & Ref. & $\begin{array}{l}\text { King } \\
\text { Are T. C. }\end{array}$ & K & & esig. \\
\hline$B$ & 10274. 06 & $50 h$ & & 9730.59 & +0.02 & $6 p^{\prime}{ }^{1} \mathrm{D}_{2}-7 s^{\prime}{ }^{3} \mathrm{D}_{1}$ & B & 7865.29 & $1 h$ & & 12710.60 & +0.11 & $6 p^{\prime}{ }^{3} \mathrm{D}_{1}^{\circ}-6 d^{\prime} \quad{ }^{1} \mathrm{P}_{1}$ \\
\hline B & 1023 & $400 h$ & -.. & 9769.41 & +0.08 & $6 p^{\prime}{ }^{3} \mathrm{~F}_{4}^{\circ}-7 s^{\prime}{ }^{3} \mathrm{D}_{3}$ & $\mathrm{D}$ & 7863.45 & $1 u$ & & 12713.57 & 10.1 & (1) \\
\hline$B$ & $\begin{array}{l}10188.24 \\
10172.1\end{array}$ & $\begin{array}{l}50 h \\
3 h\end{array}$ & -- & $\begin{array}{l}9812.55 \\
9828.1\end{array}$ & $\begin{array}{l}-0.03 \\
-0.7\end{array}$ & $\begin{array}{l}6 p^{\prime}{ }^{1} \mathrm{~F}_{3}^{0}-6 d^{\prime}{ }^{3} \mathrm{D}_{3} \\
6 p^{\prime}{ }^{3} \mathrm{P}_{2}^{\circ}-7 d d^{3}{ }^{3} \mathrm{D}_{3}\end{array}$ & $\begin{array}{l}\mathrm{C} \\
\mathrm{D}\end{array}$ & $\begin{array}{l}7839.574 \\
7828.96\end{array}$ & $\begin{array}{c}60 h \\
2\end{array}$ & -.. & $\begin{array}{l}12752.292 \\
12769.57\end{array}$ & -0.08 & $6 p^{\prime}{ }^{3} \mathrm{D}_{3}^{\circ}-6 d^{\prime} \quad{ }^{3} \mathrm{~F}_{4}$ \\
\hline B & 10146.9 & $5 H d ?$ & & 9852.5 & & & $\mathrm{~B}$ & 7798. 26 & $5 h$ & & 12819.85 & -0.01 & $6 p^{\prime}{ }^{1} \mathrm{D}_{2}^{\circ}-6 d^{\prime}{ }^{3} \mathrm{G}_{3}$ \\
\hline $\begin{array}{l}\mathrm{B} \\
\mathrm{B}\end{array}$ & 10129. 70 & 10 & $\cdots$ & 9869.25 & +0.07 & $6 p^{\prime}{ }^{1} \mathrm{D}_{2}^{\circ}-7 s^{\prime}{ }^{3} \mathrm{D}_{2}$ & D & $7783.55 ?$ & 1 & & 12844.07 & & \\
\hline$\stackrel{b}{B}$ & $\begin{array}{l}10115.2 \\
10085.5\end{array}$ & $\begin{array}{l}5 h \\
2 h\end{array}$ & $\cdots$ & $\begin{array}{l}9883.4 \\
9912.5\end{array}$ & $-0.3^{-}$ & $6 p^{\prime}{ }^{3} \mathrm{P}_{1}-6 p^{2}{ }^{3} \mathrm{P}_{2}$ & $\begin{array}{l}\mathrm{C} \\
\mathrm{B}\end{array}$ & 7780.479 & 400 & 10 & 12849.148 & +0.005 & $5 d{ }^{3} \mathrm{D}_{2}-6 p^{\prime} \quad{ }^{3} \mathrm{~F}_{2}$ \\
\hline B & 10032.10 & 200 & & 9965.27 & $\begin{array}{r}-0.0 \\
0.00\end{array}$ & $6 p{ }^{3} \mathrm{P}_{2}^{0}-5 d^{2}{ }^{3} \mathrm{P}_{1}$ & $\stackrel{B}{D}$ & $\begin{array}{l}7775.37 \\
7770.26 ?\end{array}$ & $\begin{array}{c}10 h \\
0\end{array}$ & 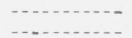 & $\begin{array}{l}12857.59 \\
12866.05\end{array}$ & +0.05 & $6 p^{\prime}{ }^{3} \mathrm{D}_{3}^{\circ}-6 d^{\prime} \quad{ }^{1} \mathrm{D}_{2}$ \\
\hline B & 10001.08 & 300 & & 9996.18 & +0.02 & $6 p^{\prime}{ }^{3} \mathrm{~F}_{3}^{0}-7 s^{\prime}{ }^{3} \mathrm{D}_{2}$ & B & 7766. 89 & $10 h l$ & & 12871.78 & -0.01 & $6 p^{\prime}{ }^{3} \mathrm{~F}_{4}^{0}-6 d^{\prime} \quad 3 \mathrm{D}_{3}$ \\
\hline B & 9830.37 & $500 h l$ & $\cdots$ & 10169.77 & -0.05 & $6 p,{ }^{1} \mathbf{P}_{\mathrm{i}}^{\circ}-7 s \quad{ }^{1} \mathrm{~S}_{0}$ & B & 7751.68 & $40 h v$ & & 12896.88 & +0.09 & $6 p^{\prime}{ }^{3} \mathrm{D}_{1}-6 d^{\prime} \quad{ }^{3} \mathrm{~F}_{2}$ \\
\hline $\begin{array}{l}\mathrm{B} \\
\mathrm{B}\end{array}$ & $\begin{array}{l}9821.60 \\
9792.91\end{array}$ & $\begin{array}{l}3 h \\
5 h\end{array}$ & & $\begin{array}{l}10178.85 \\
10208.67\end{array}$ & +0.21 & $6 p^{\prime}{ }^{3} \mathrm{D}_{\mathrm{i}}^{\circ}-6 p^{2}{ }^{1} \mathrm{D}_{2}$ & B & 7721. 78 & $10 h$ & & 12946.82 & -0.02 & $6 p^{\prime} 3 \mathrm{~F}_{3}^{\prime}-6 d^{\prime} \quad 3 \mathrm{G}_{3}$ \\
\hline $\mathrm{B}$ & 9772.62 & $6 h$ & & 10229.87 & $\begin{array}{l}-0.09 \\
+0.19\end{array}$ & $\begin{array}{l}6 p^{\prime}{ }^{3} \mathrm{P}_{2}^{0}-6 d^{\prime}{ }^{1} \mathrm{~F}_{3} \\
6 p^{\prime}{ }^{3} \mathrm{P}_{1}^{0}-6 d^{\prime}{ }^{3} \mathrm{D}_{1}\end{array}$ & $\stackrel{\mathrm{B}}{\mathrm{C}}$ & $\begin{array}{l}7706.51 \\
7672.092\end{array}$ & $\begin{array}{l}50 h v \\
600\end{array}$ & ${ }_{25 r}^{1 n}$ III & $\begin{array}{l}12972.48 \\
13030.673\end{array}$ & $\begin{array}{c}0.00 \\
-0.003\end{array}$ & \\
\hline B & 9759.35 & & & 10243.78 & -0.09 & $6 p^{\prime}{ }^{3} \mathrm{P}_{2}^{0}-6 d^{\prime}{ }^{3} \mathrm{D}_{2}$ & D & 7662.03 & 3 & & 13047.78 & & \\
\hline B & 9713.75 & 60 & & 10291.86 & +0.19 & $6 p^{\prime}{ }^{3} \mathrm{P}_{0}^{\circ}-6 d^{\prime}{ }^{3} \mathrm{D}_{1}$ & $\mathrm{~B}$ & 7642.91 & $200 h l$ & $4 n$ III & 13080.42 & 0.00 & $6 p^{\prime}{ }^{3} \mathrm{~F}_{4}^{\circ}-6 d^{\prime} \quad{ }^{3} \mathrm{G}_{5}$ \\
\hline B & 9704.42 & $20 h$ & & 10301. 76 & $\begin{array}{l}-0.02 \\
-0.08\end{array}$ & 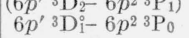 & $\begin{array}{l}\mathrm{B} \\
\mathrm{D}\end{array}$ & $\begin{array}{l}7636.90 \\
7616.55\end{array}$ & $\begin{array}{c}150 h l \\
1 u\end{array}$ & $2 n$ III & $\begin{array}{l}13090.72 \\
13125.69\end{array}$ & $\begin{array}{l}-0.18 \\
-0.32\end{array}$ & 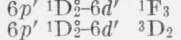 \\
\hline B & 9658.8 & $10 \mathrm{nl}$ & & 10350.4 & & & $\mathrm{C}$ & 7610.479 & 50 & III $A$ & 13136.167 & +0.013 & $5 d d^{1} \mathrm{D}_{2}-6 p^{\prime}{ }^{3} \mathrm{D}_{2}^{2}$ \\
\hline B & 9645.72 & $100 h$ & & 10364.45 & -0.11 & $6 p^{\prime}{ }^{3} \mathrm{D}_{3}^{\circ}-6 p^{2}{ }^{1} \mathrm{D}_{2}$ & B & 7575. 22 & $2 h$ & & 13197.31 & & $6 p^{\prime}{ }^{3} \mathrm{D}_{3}^{0}-6 d^{\prime} \quad 1 \mathrm{G}_{4}$ \\
\hline B & 9608.88 & 300 & & 10404.19 & -0.01 & $6 p{ }^{3} \mathrm{P}_{2}^{0}-5 d^{2}{ }^{3} \mathrm{P}_{2}$ & B & 7543.48 & $25 h$ & & 13252.84 & -0.14 & $6 p^{\prime}{ }^{3} \mathrm{~F}_{3}^{\circ}-6 d^{\prime} \quad{ }^{3} \mathrm{D}_{2}$ \\
\hline B & 9589.37 & 150 & & 10425.36 & -0.08 & $6 p{ }^{3} \mathrm{P}_{1}^{0}-5 d^{2}{ }^{1} \mathrm{D}_{2}$ & $\mathrm{~B}$ & 7528.20 & $20 h$ & & 13279.73 & -0.03 & $6 p^{\prime}{ }^{3} \mathrm{~F}_{2}^{0}-6 p^{2} \quad{ }^{1} \mathrm{D}_{2}$ \\
\hline B & 9530.30 & $10 h$ & & 10489.97 & -0.06 & $6 p^{\prime}{ }^{3} \mathrm{P}_{2}^{0}-6 d^{\prime}{ }^{3} \mathrm{~S}_{1}$ & $\mathrm{~B}$ & 7523.60 & $20 h$ & & 13287.85 & +0.04 & $6 p^{\prime}{ }^{3} \mathrm{D}_{3}^{\circ}-6 d^{\prime} \quad{ }^{3} \mathrm{P}_{2}$ \\
\hline B & 9524.70 & $60 h$ & & 10496.14 & -0.14 & $6 p^{\prime}{ }^{3} \mathrm{P}_{1}^{0}-6 d^{\prime}{ }^{3} \mathrm{D}_{2}$ & B & 7513. 40 & $3 h$ & & 13305.89 & & $6 p^{\prime}{ }^{3} \mathrm{D}_{2}^{\circ}-6 d^{\prime} \quad 1 \mathrm{D}_{2}$ \\
\hline $\mathrm{B}$ & 9455.92 & 100 & & 10572.49 & 0.00 & $6 p{ }^{3} \mathrm{P}_{1}-5 d^{2}{ }^{3} \mathrm{P}_{0}$ & C & 7488.083 & 200 & 10 & 13350.882 & -0.005 & $5 d \quad{ }^{3} \mathrm{D}_{3}-6 p^{\prime} \quad{ }^{3} \mathrm{~F}_{3}^{0}$ \\
\hline B & 9450.05 & $15 h$ & $\cdots$ & 10579.05 & +0.08 & $6 p^{\prime}{ }^{3} \mathrm{~F}_{3}^{\circ}-7 s^{\prime}{ }^{3} \mathrm{D}_{3}$ & B & 7476.21 & $30 h$ & & 13372.08 & -0.09 & $6 p^{\prime}{ }^{1} \mathrm{D}_{2}^{\circ}-6 d^{\prime}{ }^{3} \mathrm{~S}_{1}$ \\
\hline $\mathrm{B}$ & 9414.6 & $4 h$ & & 10618. 9 & +0.2 & $6 p^{\prime}{ }^{1} \mathrm{~F}_{3}^{\circ}-7 d{ }^{1} \mathrm{D}_{2}$ & B & 7459.78 & $300 h l$ & III & 13401.54 & 0.00 & $6 p^{\prime}{ }^{3} \mathrm{~F}_{3}^{0}-6 d^{\prime}{ }^{3} \mathrm{G}_{4}$ \\
\hline$\stackrel{B}{B}$ & $\begin{array}{l}9403.53 \\
9398.8\end{array}$ & $\begin{array}{l}10 \\
6 h\end{array}$ & & 10631.39 & +0.03 & $6 p^{\prime}{ }^{3} \mathrm{D}_{1}^{0}-6 p^{2}{ }^{3} \mathrm{P}_{1}$ & $\mathrm{C}$ & 7417.533 & 100 & II & 13477.866 & +0.001 & $5 d{ }^{3} \mathrm{D}_{3}-6 p^{\prime}{ }^{1} \mathrm{D}_{2}^{\circ}$ \\
\hline $\begin{array}{l}\mathrm{B} \\
\mathrm{B}\end{array}$ & 9370.06 & $\begin{array}{c}6 n \\
500\end{array}$ & & $\begin{array}{l}10636.7 \\
10669.37\end{array}$ & $\begin{array}{l}-0.4 \\
+0.09\end{array}$ & $6 p^{\prime}{ }^{3} \mathrm{D}_{3}^{\circ}-6 p^{2}{ }^{3} \mathrm{P}_{2}$ & B & 7414. 26 & $4 h l$ & & 13483.81 & +0.03 & $6 p^{\prime}{ }^{3} \mathrm{D}^{\circ}-6 d^{\prime}{ }^{3} \mathrm{P}_{0}$ \\
\hline B & 9367.45 & $80 h$ & & $\begin{array}{l}10669.37 \\
10672.34\end{array}$ & $\begin{array}{l}+0.09 \\
+0.02\end{array}$ & 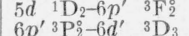 & $\mathrm{B}$ & $\begin{array}{l}7409.97 \\
7302\end{array}$ & $30 h v$ & $\begin{array}{r}1 \\
30\end{array}$ & 13491.62 & -0 . & $6 p^{\prime}{ }^{3} \mathrm{D}_{2}^{\circ}-6 d^{\prime}{ }^{3} \mathrm{P}_{1}$ \\
\hline B & 9324.58 & $100 h$ & & 10721.40 & -0.02 & $6 p^{\prime} 1 \mathrm{D}_{2}^{\circ}-7 s^{\prime} \quad 1 \mathrm{D}_{2}$ & $\mathrm{~B}$ & $\begin{array}{l}7392.411 \\
7375.59\end{array}$ & $500 \mathrm{kl}$ & $2 n$ & $\begin{array}{l}13525.668 \\
13554.50\end{array}$ & $\begin{array}{l}0.000 \\
+0.05\end{array}$ & $\begin{array}{l}6 p \\
6 p^{\prime}+1 \mathrm{D}_{2}^{\circ}-6 d^{\prime}{ }^{3} \mathrm{D}_{3}\end{array}$ \\
\hline B & 9308.08 & 100 & ... & 10740.41 & +0.08 & $6 p^{\prime}{ }^{3} \mathrm{~F}_{2}-7 s^{\prime} \quad{ }^{3} \mathrm{D}_{1}$ & $\mathrm{~B}$ & 7359.29 & 20 & III $A$ & 13584.53 & +0.05 & $5 d \quad 1 \mathrm{D}_{2}-6 p^{\prime} 3 \mathrm{D}_{3}^{3}$ \\
\hline B & 9306.52 & 10 & & 10742. 21 & -0.23 & $6 p^{\prime}{ }^{3} \mathrm{P}_{1}^{0}-6 d^{\prime} \quad{ }^{3} \mathrm{~S}_{1}$ & B & 7339.57 & $4 h$ & & 13621.03 & -0.27 & $5 d^{2}{ }^{3} \mathrm{P}_{2}-7 p^{\prime}{ }^{3} \mathrm{D}_{3}^{\circ}$ \\
\hline${ }_{\mathrm{B}}^{\mathrm{B}}$ & 9253.08 & $15 h$ &.- & 10804.25 & -0.18 & $6 p^{\prime}{ }^{3} \mathrm{P}_{0}^{\circ}-6 d^{\prime} \quad{ }^{3} \mathrm{~S}_{1}$ & B & 7326.50 & $10 h$ & & 13645.33 & -0.01 & $6 p^{\prime} 3 \mathrm{D}_{1}-6 d^{\prime} 1 \mathrm{D}_{2}$ \\
\hline $\begin{array}{l}\mathrm{B} \\
\mathrm{B}\end{array}$ & $\begin{array}{l}9245.6 \\
9219.69\end{array}$ & $100^{1 h}$ & & 10813.0 & +0.1 & $6 p^{\prime}{ }^{3} \mathrm{D}_{2}^{\circ}-6 p^{2} \quad{ }^{1} \mathrm{D}_{2}$ & D & 7319.12 & $1 u$ & & 13659.08 & & \\
\hline $\mathrm{B}$ & 9215.42 & $\begin{array}{l}100 \\
15 h\end{array}$ & & $\begin{array}{l}10843.38 \\
10848.40\end{array}$ & & $\begin{array}{ll}6 p p{ }^{3} \mathrm{P}_{1}^{\circ}-5 d^{2} & { }^{3} \mathrm{P}_{1} \\
6 p^{\prime} \mathrm{P}^{3} \mathrm{~F}_{3}^{\circ}-7 s^{\prime} & 1 \mathrm{D}_{3}\end{array}$ & $\stackrel{B}{B}$ & $\begin{array}{l}\text { 7307. } 23 \\
7304.46\end{array}$ & $\begin{array}{r}10 h \\
5 h\end{array}$ & & $\begin{array}{l}13681.31 \\
13686.50\end{array}$ & -( & $6 p^{\prime}{ }^{3} \mathrm{~F}_{3}^{\circ}-6 d^{\prime}{ }^{3} \mathrm{D}_{3}$ \\
\hline B & 9189.57 & $60 h$ & & 10878. 92 & -0.02 & $6 p^{\prime}{ }^{3} \mathrm{~F}_{2}^{0}-7 s^{\prime} \quad{ }^{3} \mathrm{D}_{2}$ & $\mathrm{C}$ & 7280.298 & 1000 & $150 r$ & 13731.924 & +0.004 & $5 d{ }^{3} \mathrm{D}_{2}-6 p^{\prime}{ }^{3} \mathrm{~F}_{3}^{\circ}$ \\
\hline B & 9159.66 & $10 h$ & $\ldots$ & 10914. 44 & +0.02 & $6 p^{\prime}, 3 \mathrm{D}_{3}-6 d^{\prime} \quad 3 \mathrm{G}_{3}$ & B & 7272.33 & $2 h$ & $\ldots$ & 13746.97 & & $6 p^{\prime}{ }^{3} \mathrm{~F}_{4}^{0}-6 d^{\prime}{ }^{3} \mathrm{~F}_{3}$ \\
\hline $\mathrm{B}$ & 9133. 29 & $15 h$ & & 10945.95 & -0.05 & $6 p^{\prime}{ }^{3} \mathrm{P}_{2}^{0}-6 d^{\prime} \quad{ }^{1} \mathrm{P}_{1}$ & $\mathrm{E}$ & 7229.56 & $10 h$ & & 13828.29 & +0.16 & $6 p^{\prime}{ }^{1} \mathrm{D}_{2}^{\circ}-6 d^{\prime}{ }^{1} \mathrm{P}_{1}$ \\
\hline B & 9101. 7 & $3 l n$ & -.. & 10983.9 & -..... & - & B & 7228.84 & $200 h l$ & 25 & 13829.67 & +0 & $6 p^{\prime}{ }^{3} \mathrm{~F}_{2}^{\circ}-6 d^{\prime}{ }^{3} \mathrm{G}_{3}$ \\
\hline B & 9097.8 & $2 h$ & & 10988.7 & & & B & 7213.56 & 10 & III $A$ & 13858.97 & +0.07 & $5 d{ }^{3} \mathrm{D}_{2}-6 p^{\prime}{ }^{1} \mathrm{D}_{2}^{\circ}$ \\
\hline B & 9018.63 & $3 h$ & $\ldots$ & 11085.12 & -0.29 & $6 p^{\prime}{ }^{3} \mathrm{D}_{2}^{\circ}-6 p^{2} \quad{ }^{3} \mathrm{P}_{2}$ & B & 7208.18 & $20 h$ & III & 13869.31 & +0.15 & $6 p^{\prime}{ }^{3} \mathrm{~F}_{2}^{0}-6 d^{\prime}{ }^{3} \mathrm{D}_{1}$ \\
\hline B & 8975.6 & $2 h$ & & 11138.3 & -0.6 & $6 p^{\prime}{ }^{3} \mathrm{P}_{2}^{\circ}-9 \varsigma \quad{ }^{3} \mathrm{~S}_{1}$ & C & 7195.235 & 200 & 80 & 13894.263 & 0.000 & $6 p{ }^{3} \mathrm{P}_{0}^{0}-78{ }^{3} \mathrm{~S}_{1}$ \\
\hline B & 8937.93 & $10 h$ & ... & 11185. 20 & -0.25 & $6 p^{\prime}{ }^{3} \mathrm{D}_{3}^{\circ}-6 d^{\prime} \quad{ }^{1} \mathrm{~F}_{3}$ & B & 7153.54 & $80 h v$ & 5 & 13975.25 & +0.10 & $6 p^{\prime}{ }^{3} \mathrm{~F}_{4}^{\circ}-6 d^{\prime}{ }^{3} \mathrm{~F}_{4}$ \\
\hline B & 8927.41 & $7 h l$ & & 11198. 39 & -0.02 & $6 p^{\prime}{ }^{3} \mathrm{P}_{1}^{\circ}-6 d^{\prime} \quad{ }^{1} \mathrm{P}_{1}$ & B & 7133.16 & $4 h$ & $\cdots$ & 14015.17 & -0.27 & $5 d^{2}{ }^{1} \mathrm{D}_{2}-7 p^{\prime}{ }^{1} \mathrm{D}_{2}$ \\
\hline $\mathrm{B}$ & 8914.99 & 150 & -.. & 11213.99 & 0.00 & $6 p{ }^{3} \mathrm{P}_{0}^{0}-5 d^{2} \quad{ }^{3} \mathrm{P}_{1}$ & $\mathrm{D}$ & 7129.56 & 2 & & 14022. 24 & & \\
\hline B & 8909.83 & $3 h$ & $\cdots$ & 11220.48 & -0.08 & $6 p^{\prime}{ }^{3} \mathrm{D}_{3}^{\circ}-6 d^{\prime} \quad{ }^{3} \mathrm{D}_{2}$ & B & 7126.60 & $10 h$ & & 14028.08 & & \\
\hline $\mathrm{B}$ & 8860.98 & 100 & -- & 11282. 33 & +0.01 & $6 p{ }^{3} \mathrm{P}_{1}^{\circ}-5 d^{2} \quad{ }^{3} \mathrm{P}_{2}$ & $\mathrm{C}$ & 7120.329 & $800 h v$ & 200 & 14040.431 & 0.000 & $5 d{ }^{3} \mathrm{D}_{1}-6 p^{\prime}{ }^{1} \mathrm{D}_{2}^{\circ}$ \\
\hline $\begin{array}{l}\mathrm{B} \\
\mathrm{B}\end{array}$ & $\begin{array}{l}8799.76 \\
8793.36\end{array}$ & $\begin{array}{r}100 h \\
3 h\end{array}$ & $\cdots$ & 11360. & +0.02 & $6 p^{\prime}{ }^{1} \mathrm{~F}_{3}^{0}-6 d^{\prime} \quad{ }^{1} \mathrm{G}_{4}$ & D & 7110.24 & $1 u$ & $\ldots$ & 14060.35 & $\ldots-\ldots$ & - \\
\hline $\mathrm{B}$ & 8767.7 & $\begin{array}{l}5 n \\
1 h\end{array}$ & & $\begin{array}{l}11369.10 \\
11402.4\end{array}$ & $\begin{array}{l}-0.02 \\
+0.1\end{array}$ & $\begin{array}{ll}6 p^{\prime} \\
6 p^{\prime} 3 \mathrm{D}_{2}^{\circ}-6 d^{\prime}\end{array}$ & $\begin{array}{l}\mathrm{D} \\
\mathrm{B}\end{array}$ & $\begin{array}{l}7097.99 \\
7090.01\end{array}$ & $100 h l$ & $5 n$ III & $\begin{array}{l}14084.61 \\
14100.47\end{array}$ & & $6 p^{\prime} 3 \mathrm{~F}_{0}^{0}-6 d^{\prime} 1 \mathrm{~F}_{3}$ \\
\hline B & 8737.74 & $3 h$ & & 11441.47 & & $0 p-D_{2}-0 a-D_{1}$ & B & 7072.38 & $1 \mathrm{~h}$ & 111 & $\begin{array}{l}14100.47 \\
14135.62\end{array}$ & $\begin{array}{l}-0.18 \\
-0.14\end{array}$ & $\begin{array}{ll}6 p^{\prime} \\
6 p^{\prime}{ }^{3} \mathrm{~F}_{2}^{2}-6 d^{\prime}-3 \mathrm{D}_{2}\end{array}$ \\
\hline B & 8710.82 & $3 h$ & $\ldots$ & 11476.83 & -.... & $\ldots$ & B & 7069.43 & 3 & & 14141.52 & $\begin{array}{l}-0.14 \\
+0.11\end{array}$ & $6 p^{\prime} 3 \mathrm{~F}_{3}^{0}-6 d^{\prime}{ }^{3} \mathrm{~F}_{2}$ \\
\hline D & 8659.30 & 1 & ... & 11545.11 & & & $\mathrm{C}$ & 7059.941 & 2000 & $400 r$ & 14160.526 & 0.000 & $5 d{ }^{3} \mathrm{D}_{3}-6 p^{\prime}{ }^{3} \mathrm{~F}_{4}^{2}$ \\
\hline $\mathrm{B}$ & 8654.07 & 60 & -.. & 11552.09 & +0.03 & $5 d \quad{ }^{1} \mathrm{D}_{2}-6 p^{\prime} \quad{ }^{3} \mathrm{~F}_{3}^{\circ}$ & $\mathrm{B}$ & 6986.79 & 5 & & 14308.78 & +0.02 & $5 d \quad{ }^{1} \mathrm{D}_{2}-6 p^{\prime}{ }^{3} \mathrm{P}_{1}^{0}$ \\
\hline B & 8593.48 & $4 h l$ & & 11633.54 & -0.24 & $6 p^{\prime}{ }^{3} \mathrm{D}_{2}^{0}-6 d^{\prime} \quad{ }^{1} \mathrm{~F}_{3}$ & B & 6961.63 & $2 h$ & & 14360.50 & -0.04 & $6 p^{\prime}{ }^{1} \mathrm{D}_{2}^{0}-7 d{ }^{1} \mathrm{D}_{2}$ \\
\hline B & 8581.98 & $50 h l$ & $\ldots$ & 11649.13 & +0.12 & $6 p^{\prime}{ }^{3} \mathrm{D}_{3}^{\circ}-6 d^{\prime} \quad{ }^{3} \mathrm{D}_{3}$ & D & 6951.35 & $1 u$ & $\ldots$ & 14381.73 & -0.19 & $6 p^{\prime}{ }^{3} \mathrm{~F}_{2}^{\circ}-6 d^{\prime}{ }^{3} \mathrm{~S}_{1}$ \\
\hline D & $\begin{array}{l}8569 . \\
8567\end{array}$ & $1 r$ & & 11665.42 & & $\mathrm{a}=30^{\circ} \mathrm{de}$ & B & 6932. 92 & $2 h$ & $\cdots$ & 14419.96 & -0.06 & $6 p^{\prime}{ }^{3} \mathrm{~F}_{4}^{\circ}-6 d^{\prime}{ }^{1} \mathrm{G}_{4}$ \\
\hline $\begin{array}{l}\mathrm{B} \\
\mathrm{B}\end{array}$ & $\begin{array}{l}8567.58 \\
8559.97\end{array}$ & $\begin{array}{l}40 h l \\
600 h\end{array}$ & 4 & $\begin{array}{l}11668.70 \\
11679.08\end{array}$ & $\begin{array}{l}-0.19 \\
+0.05\end{array}$ & $\begin{array}{ll}6 p^{\prime} 3 \mathrm{D}_{2}^{\circ}-6 d^{\prime} & 3 \mathrm{D}_{2} \\
5 d_{2}-6 n^{\prime} & \end{array}$ & $\mathrm{D}$ & 6874.16 & $\begin{array}{r}1 u \\
100 h\end{array}$ & $8 n$ & 14543. 22 & & $6 n^{\prime} 3 \mathrm{~F}^{\circ}-6 d^{\prime} 3 \mathrm{~F}$ \\
\hline D & 8542.37 & $1 u$ & & 11703.14 & & 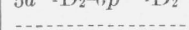 & $\mathrm{C}$ & $\begin{array}{l}6867.85 \\
6865.692\end{array}$ & $\begin{array}{l}100 h \\
200\end{array}$ & 30 & $\begin{array}{l}14556.59 \\
14561.164\end{array}$ & $\begin{array}{l}+0.01 \\
-0.003\end{array}$ & $5 d \quad 1 \mathrm{D}_{2}-6 p^{\prime}{ }^{3} \mathbf{P}_{2}^{0}$ \\
\hline B & 8521.96 & $8 h l$ & & 11731. 17 & -0.01 & $6 p^{\prime}{ }^{3} \mathrm{~F}_{2}^{0}-7 s^{\prime} \quad{ }^{1} \mathrm{D}_{2}$ & B & 6771.85 & $60 \mathrm{~h}$ & $2 n$ III & 14762.95 & -0.03 & $6 p^{\prime}{ }^{1} \mathrm{D}_{2}^{\circ}-6 d^{\prime}{ }^{1} \mathrm{D}_{2}$ \\
\hline B & 8514. 23 & $30 h l$ & -.- & 11741.82 & +0.05 & $6 p^{\prime}{ }^{3} \mathrm{D}_{1}^{\circ}-6 d^{\prime}{ }^{3} \mathrm{D}_{1}$ & D & 6769.60 & $2 u$ & - n & 14767.84 & & \\
\hline B & 8414.58 & $4 h v$ & $\ldots$ & 11880.87 & +0.12 & $6 p^{\prime}{ }^{3} \mathrm{P}_{2}^{0}-6 d^{\prime} \quad{ }^{1} \mathrm{D}_{2}$ & B & 6761.86 & $2 h$ & & 14784.76 & -0.03 & $6 p^{\prime}{ }^{3} \mathrm{~F}_{3}^{0}-6 d^{\prime}{ }^{3} \mathrm{~F}_{4}$ \\
\hline B & 8350.76 & $2 h$ & $\cdots$ & 11971.67 & -0.03 & $6 p^{\prime}{ }^{3} \mathrm{P}_{\mathrm{i}}-6 d^{\prime} \quad{ }^{3} \mathrm{P}_{0}$ & B & 6714.03 & $2 h$ & & 14890.08 & +0.12 & $6 p^{\prime}{ }^{3} \mathrm{~F}_{3}^{\circ}-6 d^{\prime}{ }^{1} \mathrm{D}_{2}$ \\
\hline $\mathrm{D}$ & 8328.82 & $3 u$ & $\cdots$ & 12003. 20 & & & $\mathrm{C}$ & 6693.842 & 600 & 70 & 14934.987 & +0.002 & $5 d{ }^{3} \mathrm{D}_{3}-6 p^{\prime}{ }^{3} \mathrm{D}_{2}^{\circ}$ \\
\hline B & 8325.38 & $20 h$ & & 12008. 27 & -0.10 & $6 p^{\prime}{ }^{3} \mathrm{D}_{1}^{\mathrm{i}}-6 d^{\prime} \quad{ }^{3} \mathrm{D}_{2}$ & C & 6675.271 & 500 & & 14976.538 & -0.001 & $5 d{ }^{3} \mathrm{D}_{2}-6 p^{\prime} 3 \mathrm{D}_{1}^{\circ}$ \\
\hline $\mathrm{D}$ & 8288.10 & $1 u$ & $\cdots$ & 12062.18 & & & C & 6654.102 & 50 & 10 & 15024.183 & 0.000 & $6 p^{\prime}{ }^{3} \mathrm{~F}_{2}^{0}-6 d^{\prime}{ }^{3} \mathrm{~F}_{2}$ \\
\hline$\stackrel{B}{B}$ & $\begin{array}{l}8285 . \\
8264\end{array}$ & $6 n v$ & & 12066. 69 & +0.02 & $6 p^{\prime}{ }^{3} \mathrm{P}^{\circ}-6 d^{\prime}$ & F & & & $1 n$ & 15050.64 & & \\
\hline B & $\begin{array}{l}8204.01 \\
8224.41\end{array}$ & $\begin{array}{l}\text { sh } \\
5 h\end{array}$ & (n) & 12097. 34 & & $6 p^{\prime}{ }^{3} \mathrm{D}_{2}^{\circ}-6 d^{\prime}$ & $\mathrm{C}$ & 6595.326 & 1000 & & 15158.075 & +0.003 & $5 d{ }^{3} \mathrm{D}_{1}-6 p^{\prime}{ }^{3} \mathrm{D}_{1}^{\circ}$ \\
\hline C & 8210.239 & $300 h$ & 4 III? & $\begin{array}{l}12155.59 \\
12176.570\end{array}$ & & $6 n 1 \mathrm{P}^{\circ}$ & $B$ & & $20 h$ & & & -..... & - \\
\hline B & 8161.58 & $10 h l$ & n. & 12249.17 & & $0 p-\Gamma=0$ & $\mathrm{~B}$ & $\begin{array}{l}0505.41 \\
6564.33\end{array}$ & $3 h$ & & $\begin{array}{l}15225.98 \\
15229.65\end{array}$ & & \\
\hline B & 8158.12 & $3 h$ & $\ldots$ & 12254. 36 & -0.17 & $6 p^{\prime} 3 \mathrm{D}_{1}-6 d^{\prime}{ }^{3} \mathrm{~S}_{1}$ & $\mathrm{D}$ & 6544. & 1 & & & & \\
\hline B & 8147.78 & $30 h$ & & 12269.91 & -0.10 & $6 p^{\prime}{ }^{1} \mathrm{D}_{2}^{\circ}-6 p^{2} \quad{ }^{1} \mathrm{D}_{2}$ & $\mathrm{C}$ & 6527.312 & $(50 \mathrm{R})$ & 250 & 15316.020 & +0.002 & $5 d \mathrm{D}_{2}-6 p^{\prime} \mathrm{D}_{2}$ \\
\hline B & 8120.49 & $30 h v$ & & 12311.15 & +0.03 & $6 p^{\prime}{ }^{3} \mathrm{P}_{2}^{0}-6 d^{\prime} \quad{ }^{3} \mathrm{P}_{2}$ & C & 6498.759 & $(100 \mathrm{R})$ & $300 r$ & 15383.311 & +0.003 & $5 d{ }^{3} \mathrm{D}_{3}-6 p^{\prime} 3 \mathrm{D}_{3}^{3}$ \\
\hline B & & $1 h$ & -... & 12381.14 & +0.08 & $6 p^{\prime}{ }^{3} \mathrm{P}_{0}^{\circ}-6 d^{\prime} \quad{ }^{3} \mathrm{P}_{1}$ & $\mathrm{C}$ & 6482.908 & $(50 \mathrm{R})$ & 200 & 15420.924 & +0.013 & $5 d \quad 1 \mathrm{D}_{2}-6 p^{\prime}{ }^{1} \mathrm{~F}_{3}^{\circ}$ \\
\hline B & 8018. 23 & 10 & -... & 12468.16 & +0.05 & $5 d{ }^{3} \mathrm{D}_{3}-6 p^{\prime}{ }^{3} \mathrm{~F}_{2}^{\circ}$ & C & 6450.854 & $(30)$ & 125 & .15497 .549 & -0.002 & $5 d{ }^{3} \mathrm{D}_{1}-6 p^{\prime}{ }^{3} \mathrm{D}_{2}^{\circ}$ \\
\hline B & 7982. 40 & 15 ho & -- - & 12524.12 & -0.04 & $6 p^{\prime} 3 \mathrm{D}_{3}^{\circ}-6 d^{\prime} \quad{ }^{3} \mathrm{~F}_{3}$ & G & 6411.74 & 1 & III $A$ & 15592.08 & & \\
\hline B & 7961.24 & $10 h v$ & .... & 12557.41 & +0.10 & $6 p^{\prime}{ }^{3} \mathrm{D}_{2}^{2}-6 d^{\prime} \quad{ }^{3} \mathrm{~F}_{2}$ & $\mathrm{E}$ & 6411.150 & 1 & & 15593.52 & +0.06 & $6 p{ }^{3} \mathrm{P}_{\mathrm{i}}-7 s \quad{ }^{1} \mathrm{~S}_{0}$ \\
\hline B & 7957.33 & $2 h$ & $\cdots$ & 12563.58 & +0.05 & $6 p^{\prime}{ }^{3} \mathrm{P}_{1}^{\circ}-f i d^{\prime} \quad{ }^{3} \mathrm{P}_{2}$ & $\mathrm{E}$ & 6353.200 & 1 & & 15735.75 & & $6 p{ }^{1} \mathrm{P}_{1}^{\circ}-7 \mathrm{~s}^{\prime}{ }^{1} \mathrm{D}_{2}$ \\
\hline B & 7939.42 & $3 h$ & & 12591.92 & +0.02 & $6 p^{\prime}{ }^{3} \mathrm{~F}_{4}^{0}-6 d^{\prime}{ }^{3} \mathrm{G}_{4}$ & C & 6341.682 & $(50)$ & 150 & 15764.338 & -0.003 & $5 d{ }^{3} \mathrm{D}_{2}-6 p^{\prime} 3 \mathrm{D}_{3}^{\circ}$ \\
\hline C & 7911.338 & 200 & $3 \quad 1 A$ & 12636.616 & 0.000 & $6 s^{2}{ }^{1} \mathrm{~S}_{0}-6 p \quad{ }^{3} \mathrm{P}_{1}$ & G & 6323.4 & 1 & III? & 15809.90 & -..... & - \\
\hline $\mathrm{B}$ & $\begin{array}{l}7905.751 \\
7877.93\end{array}$ & 500 & $6 \mathrm{I}$ & 12645.546 & 0.000 & $6 p{ }^{3} \mathrm{P}_{2}^{\circ}-7.9$ & D & 6300.30 & $2 u$ & $\mathrm{Tix}^{-1}$ & 15867.87 & & \\
\hline D & 1076.90 & 2011 & & 12690.20 & -0.20 & $6 p{ }^{1} \mathrm{P}_{\mathrm{i}}^{\circ}-6 d$ & G & 6235.3 & $2 u$ & $30 n$ & 16033.28 & & \\
\hline
\end{tabular}


TABLE 2-Continued

\begin{tabular}{|c|c|c|c|c|c|c|c|c|c|c|c|c|c|c|}
\hline \multirow{2}{*}{$\begin{array}{l}\text { Lit. } \\
\text { Ref. }\end{array}$} & \multirow{2}{*}{ I. A. } & & tensity & & & & & & & tensity & & & & \\
\hline & & Ref. & $\begin{array}{l}\text { King } \\
\text { Are T. C. }\end{array}$ & 10 & $0-\mathrm{c}$ & & & & Ref. & $\begin{array}{c}\text { King } \\
\text { Are T. } \mathrm{C} .\end{array}$ & $\pi$ & & & Desig. \\
\hline D & 6174.54 & 4 & & 16191.06 & & & $\mathrm{H}$ & 4724.742 & 3 & 3 III & 21159.26 & +0.04 & $6 p$ & ${ }^{3} \mathrm{P}_{1}^{\circ}-7 s^{\prime} \quad 1 \mathrm{D}_{2}$ \\
\hline $\mathrm{D}$ & 6140.39 & 5 & & 6281.11 & & & $\mathrm{C}$ & 4700.427 & (3) & 20 III & 21268. 729 & -0.004 & $6 p$ & ${ }^{3} \mathrm{P}_{1}-8 s \quad 3 \mathrm{~S}_{1}$ \\
\hline $\mathrm{H}$ & 6129. 335 & $3 u$ & 3 III & & -0.05 & $6 p \quad{ }^{1} \mathrm{P}_{\mathrm{j}}-6 p^{2}{ }^{1} \mathrm{~S}_{0}$ & F & 4699.108 & $-\cdots$ & 15 NIII & 21274.68 & 0.00 & $6 p$ & ${ }^{1} \mathrm{P}_{1}^{0}-9 d \quad{ }^{1} \mathrm{D}_{2}$ \\
\hline$\stackrel{C}{G}$ & $\begin{array}{l}6110.784 \\
6086.4\end{array}$ & $\begin{array}{c}(100 \mathrm{R}) \\
1 u\end{array}$ & $300 r \quad$ II & $\begin{array}{l}16359.994 \\
16425.53\end{array}$ & $\begin{array}{l}-0.004 \\
+0.09\end{array}$ & $\begin{array}{l}5 d{ }^{3}{ }^{3} \mathrm{D}_{3}-6 p^{\prime}{ }^{3} \mathrm{P}_{2}^{\circ} \\
5 d^{2}{ }^{3} \mathrm{P}_{1}-39905^{\circ}\end{array}$ & $\stackrel{I}{\mathrm{C}}$ & $\begin{array}{l}4698.10 \\
4691.617\end{array}$ & $\begin{array}{c}2 h \\
(8)\end{array}$ & 35 II & $\begin{array}{l}21279.25 \\
21308.667\end{array}$ & -0.015 & $\overline{6 p}$ & ${ }^{3} \mathrm{P}_{2}^{0}-6 p^{2} 3 \mathrm{P}_{1}$ \\
\hline $\mathrm{C}$ & 6083.402 & (5) & $5 \operatorname{III} A$ & 16433. 632 & -0.002 & $6 p{ }^{1} \mathrm{P}_{1}^{0}-6 p^{2}{ }^{3} \mathrm{P}_{0}$ & $J$ & 4674.97 & & & 21384.52 & & & \\
\hline $\mathrm{C}$ & 6063.117 & $(50 \mathrm{R})$ & 200 & 16488.612 & -0.010 & $5 d{ }^{3} \mathrm{D}_{2}-6 p^{\prime}{ }^{3} \mathrm{P}_{\mathrm{i}}^{\circ}$ & C & 4673. 619 & (3) & II & 21390.727 & +0.001 & $5 d$ & ${ }^{3} \mathrm{D}_{3}-7 p \quad{ }^{3} \mathrm{P}_{2}^{\circ}$ \\
\hline $\begin{array}{l}\mathrm{D} \\
\mathrm{C}\end{array}$ & $\begin{array}{l}6035.52 \\
6019.470\end{array}$ & $\begin{array}{c}1 u \\
(50)\end{array}$ & 100 II & $\begin{array}{l}16563.99 \\
16608.170\end{array}$ & -0.002 & & I & $\begin{array}{l}4670.75 \\
4668.56\end{array}$ & $\begin{array}{l}1 h \\
1 h\end{array}$ & & $\begin{array}{l}21403.85 \\
21413.89\end{array}$ & -...... & & \\
\hline$\stackrel{\mathrm{C}}{\mathrm{C}}$ & 5997. 088 & $(50)$ & 100 II & 16670.153 & $\begin{array}{l}-0.002 \\
-0.002\end{array}$ & $\begin{array}{l}5 d d^{3} \mathrm{D}_{1}-6 p^{\prime} 3 \mathrm{P}_{0}^{0} \\
5 d{ }^{3} \mathrm{D}_{1}-6 p^{\prime} 3 \mathrm{P}_{1}^{0}\end{array}$ & G & $\begin{array}{l}4008.50 \\
4662.8\end{array}$ & $1 u$ & 10 NIV? & $\begin{array}{l}21413.89 \\
21440.34\end{array}$ & - nes & & \\
\hline G & 5985.2 & $1 u$ & & 16703.25 & 0.00 & $5 d^{2} 1 \mathrm{D}_{2}-39765^{\circ}$ & G & 4651.8 & $1 u$ & $5 N I V ?$ & 21491.04 & - n..... & & \\
\hline $\mathrm{H}$ & 5978. 496 & 2 & $\operatorname{III} A$ & 16721.98 & -0.10 & $6 p{ }^{3} \mathrm{P}_{2}^{0}-6 d{ }^{1} \mathrm{D}_{2}$ & $\mathrm{H}$ & 4642.038 & $2 r$ & 4NIV? & 21536. 23 & & & \\
\hline $\mathrm{C}$ & 5971. 699 & 50 & 100 II & 16741.027 & -0.004 & $5 d{ }^{3} \mathrm{D}_{2}-6 p^{\prime}{ }^{3} \mathrm{P}_{2}^{\circ}$ & $\mathrm{H}$ & 4636.333 & $3 r$ & $15 \mathrm{NIV} ?$ & 21562.73 & $\ldots$ & & \\
\hline $\mathrm{H}$ & $\begin{array}{l}5964.787 \\
5962.445\end{array}$ & $\begin{array}{l}2 \\
2\end{array}$ & $\begin{array}{ll}5 & \text { III } \\
2 & \text { III } A\end{array}$ & $\begin{array}{l}16760.42 \\
16767.00\end{array}$ & -.... & - n & $\stackrel{J}{C}$ & $\begin{array}{l}4629.63 \\
4628.328\end{array}$ & $(3)^{--}$ & $25 n$ III & $\begin{array}{l}21593.95 \\
21600.044\end{array}$ & & & $\mathrm{D}_{2-7 p}{ }^{3} \mathrm{p}_{\mathrm{i}}^{0}$ \\
\hline $\begin{array}{l}\mathrm{H} \\
\mathrm{D}\end{array}$ & $\begin{array}{l}5962.445 \\
5935.43\end{array}$ & $\frac{2}{1 u}$ & $\begin{array}{c}2 \quad 111 A \\
-\end{array}$ & $\begin{array}{l}10808.00 \\
16843.31\end{array}$ & -0.08 & $5 d^{2} 1 \mathrm{D}_{2}-39905^{\circ}$ & I & $\begin{array}{l}4628.328 \\
4624.21\end{array}$ & $\begin{array}{l}(3) \\
1 h\end{array}$ & $25 n \mathrm{II}$ & $\begin{array}{l}21600.044 \\
21619.26\end{array}$ & 0.000 & $5 d$ & ${ }^{3} \mathrm{D}_{2}-7 p \quad{ }^{3} \mathrm{P}_{1}^{\mathrm{i}}$ \\
\hline G & 5927.7 & 1 & & 16865. 28 & & & $\mathrm{C}$ & 4619. 924 & $(3)$ & $20 n$ III & 21639. 336 & +0.008 & $6 p$ & ${ }^{3} \mathbf{P}_{0}^{0}-8 s{ }^{3 \mathrm{~S}_{1}}$ \\
\hline $\mathrm{C}$ & 5907.639 & (10) & II $A$ & 16922.558 & -0.006 & $5 d{ }^{3} \mathrm{D}_{1}-6 p^{\prime}{ }^{3} \mathrm{P}_{2}^{\circ}$ & $\mathbf{J}$ & 4606. & & 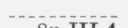 & 21702. 94 & & & \\
\hline$\stackrel{G}{G}$ & $\begin{array}{l}5859.29 \\
5849.28\end{array}$ & $\begin{array}{l}1 \\
1\end{array}$ & (n) & $\begin{array}{l}17062.19 \\
17091.38\end{array}$ & -...- & - & $\stackrel{\mathrm{C}}{\mathrm{H}}$ & $\begin{array}{l}4604.982 \\
4599.751\end{array}$ & $\stackrel{(1)}{6 R}$ & $\begin{array}{c}8 n \operatorname{III} A \\
30\end{array}$ & $\begin{array}{l}21709.548 \\
21734.22\end{array}$ & $\begin{aligned} & 0.000 \\
+ & 0.06\end{aligned}$ & $\begin{array}{l}5 d \\
6 p\end{array}$ & $\begin{array}{ll}{ }^{3} \mathrm{D}_{1}-7 p & { }^{3} \mathrm{P}_{0}^{0} \\
{ }^{3} \mathrm{P}_{1}^{*}-6 p^{2} & { }^{1} \mathrm{~S}_{0}\end{array}$ \\
\hline $\mathrm{C}$ & 5826. 277 & $(100)$ & 150 & 17158.874 & -0.001 & $5 d{ }^{1} \mathrm{D}_{2}-6 p^{\prime}{ }^{1} \mathrm{P}_{1}$ & J & 4593. 16 & & & 21765.40 & & & \\
\hline $\mathrm{C}$ & 5818.831 & (3) & III $A$ & 17180.831 & -0.025 & $6 p{ }^{3} \mathrm{P}_{2}^{0}-6 d{ }^{3} \mathrm{D}_{1}$ & $\mathrm{C}$ & 4591.824 & (2) & $10 n$ III & 21771. 758 & -0.001 & $5 d$ & ${ }^{3} \mathrm{D}_{2}-7 p \quad \mathrm{P}_{2}^{\circ}$ \\
\hline G & $\begin{array}{l}5818.25 \\
5805.685\end{array}$ & $\begin{array}{c}1 \\
(10)\end{array}$ & 20 II & $\begin{array}{l}17182.53 \\
17219.734\end{array}$ & -0.008 & $5 d{ }^{3} \mathrm{D}_{3}-6 p^{\prime} 1 \mathrm{~F}_{3}^{\circ}$ & $\underset{\mathrm{C}}{\mathrm{J}}$ & $\begin{array}{l}4591.07 \\
4589.754\end{array}$ & $\overline{(2)}$ & $8 n$ II & $\begin{array}{l}21775.31 \\
21781.577\end{array}$ & .000 & $5 d$ & $3 \mathrm{D}_{1}-7 p^{3} \mathrm{P}_{1}$ \\
\hline $\mathrm{C}$ & 5800.229 & $(100)$ & 100 III & 17235. 932 & $\begin{array}{r}0.000 \\
+0.001\end{array}$ & $6 p{ }^{3} \mathrm{P}_{2}^{0}-6 d \quad 3 \mathrm{D}_{2}$ & $\mathrm{C}$ & 4579.639 & (15) & 80 II & 21829.685 & 0.000 & $6 p$ & ${ }^{3} \mathrm{P}_{2}^{\circ}-6 p^{2}{ }^{1} \mathrm{D}_{2}$ \\
\hline $\mathrm{H}$ & 5784.105 & $2 u$ & 4 III & 17283. 97 & -0.19 & $6 p \quad{ }^{1} \mathrm{P}_{1}^{0}-6 p^{2} \quad 1 \mathrm{D}_{2}$ & $\mathrm{C}$ & 4573.854 & (10) & 40 & 21857.293 & +0.011 & $6 p$ & ${ }^{3} \mathrm{P}_{1}^{0}-6 p^{2}{ }^{3} \mathrm{P}_{0}$ \\
\hline $\mathrm{C}$ & 5777. 622 & $(500)$ & $400 r \quad$ II & 17303. 372 & 0.000 & $6 p{ }^{3} \mathrm{P}_{2}^{0}-6 d{ }^{3} \mathrm{D}_{3}$ & I & 4562.75 & 1 & - & 21910.47 & & & \\
\hline $\begin{array}{l}\mathrm{H} \\
\mathrm{H}\end{array}$ & $\begin{array}{l}5720.712 \\
5718.364\end{array}$ & $\begin{array}{l}1 \\
1\end{array}$ & - & $\begin{array}{l}17475.49 \\
17482.67\end{array}$ & -....... & - & $\stackrel{\mathrm{C}}{\mathrm{K}}$ & $\begin{array}{l}4523.171 \\
4512.89\end{array}$ & $\begin{array}{c}(10) \\
1\end{array}$ & $60 n$ & $\begin{array}{l}22102.206 \\
22152.54\end{array}$ & -0.003 & $6 p$ & $-6 p^{2}{ }^{3} \mathrm{P}_{2}$ \\
\hline $\mathrm{H}$ & 5715. 953 & 1 & $\cdots$ & 17490.04 & -... & & $\mathrm{C}$ & 4505.924 & (10) & 40 & 22186.803 & -0.001 & $6 p$ & ${ }^{3} \mathrm{P}_{1}-6 p^{2}{ }^{3} \mathrm{P}_{1}$ \\
\hline $\mathrm{H}$ & 5713.554 & 2 & $4 n \mathrm{III}$ & 17497.39 & & & $\mathrm{~K}$ & 4504.36 & $1 n$ & & 22194.49 & -0 & $6 p$ & ${ }^{3} \mathrm{P}_{2}^{0}-7 d \quad 3 \mathrm{D}_{1}^{1}$ \\
\hline $\bar{H}$ & 709.546 & 1 & $2 n \mathrm{III}$ & 17509.67 & $\ldots$ & & $\mathrm{C}$ & 4493. 637 & $(6 v)$ & $50 n$ & 22247. 469 & -0.004 & $6 p$ & ${ }^{3} \mathrm{P}_{2}^{0}-7 d \quad{ }^{3} \mathrm{D}_{2}$ \\
\hline $\mathrm{H}$ & 5706.042 & 1 & - n & 17520.42 & & $\ldots$ & $\mathrm{C}$ & 4488.977 & $(8 v)$ & $60 n$ & 22270.564 & 0. & $6 p$ & ${ }^{3} \mathrm{P}_{2}^{0}-7 d{ }^{3} \mathrm{D}_{3}$ \\
\hline H & 5704.820 & 1 & & 17524.17 & & & $\mathrm{C}$ & 4467.093 & (3) & III & 22379.664 & +0.1 & $6 p$ & ${ }^{3} \mathrm{P}_{2}^{0}-6 d^{\prime}{ }^{3} \mathrm{G}_{3}$ \\
\hline C & 5680.184 & $\ldots$ & $10 \operatorname{III} A$ & 17600.192 & -0.007 & $6 p{ }^{3} \mathrm{P}_{1}-6 d \quad{ }^{1} \mathrm{D}_{2}$ & K & 4459.18 & 0 & & 22419.36 & +0.2 & $6 p$ & ${ }^{3} \mathrm{P}_{2}^{\circ}-6 d^{\prime}{ }^{3} \mathrm{D}_{1}$ \\
\hline $\mathrm{C}$ & 5679.997 & & $2 \operatorname{III} A$ & 17600. 771 & -0.004 & $5 d{ }^{3} \mathrm{D}_{2}-6 p^{\prime}{ }^{1} \mathrm{~F}_{3}^{0}$ & C & 4431.894 & (10) & 40 & 22557.403 & +0.004 & $6 p$ & ${ }^{3} \mathrm{P}_{0}^{0}-6 p^{2}{ }^{3} \mathrm{P}_{1}$ \\
\hline I & 5664.3 & $1 h$ & & 17649.53 & +0.49 & $6 p \quad{ }^{1} \mathrm{P}_{1}^{0}-7 d \quad{ }^{3} \mathrm{D}_{1}$ & K & 4430.33 & 1 & & 22565.35 & & & \\
\hline G & 5641.1 & $1 u$ & - . & 17722.12 & ........ & - & $\mathrm{C}$ & 4413. 664 & (2) & III & 22650.573 & 0.000 & $6 p$ & ${ }^{3} \mathrm{P}_{2}^{0}-6 d^{\prime}{ }^{1} \mathrm{~F}_{3}$ \\
\hline $\mathrm{H}$ & 628. 938 & $1 U$ & - n & 17760.41 & +008 & $5 d^{2} 3 \mathrm{P}-12 f^{3} \mathrm{~F}_{0}^{0}$ & H & 4406. 846 & $\begin{array}{r}4 r \\
(20)\end{array}$ & III & $\begin{array}{l}22685.59 \\
20707.808\end{array}$ & $\begin{array}{l}-0.09 \\
+0.001\end{array}$ & $\begin{array}{l}6 p \\
6 p\end{array}$ & 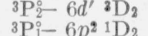 \\
\hline $\begin{array}{l}\mathrm{H} \\
\mathrm{G}\end{array}$ & $\begin{array}{l}5625.701 \\
5620.4\end{array}$ & $\begin{array}{l}1 U \\
1 u\end{array}$ & $20 n$ IV? & $\begin{array}{l}17770.63 \\
17787.39\end{array}$ & +0. & $5 d^{2} \mathrm{P}_{1}-12 f \mathrm{~F}_{2}$ & I & $\begin{array}{l}4402.539 \\
4375.88\end{array}$ & ${ }^{(20)} \mathrm{H}$ & 60 & $\begin{array}{l}22707.808 \\
22846.13\end{array}$ & +0.001 & $\sigma p$ & ${ }_{1}-6 p^{2} D_{2}$ \\
\hline $\mathrm{G}$ & 5620.0 & $1 u$ & $4 n$ IV? & 17788. 66 & -.. & $\ldots$ & $\stackrel{1}{C}$ & 4359.527 & (2) & III & 22931.845 & 0.000 & $6 p$ & ${ }^{3} \mathrm{P}_{2}^{0}-6 d^{\prime} \quad 3 \mathrm{~S}_{1}$ \\
\hline $\mathrm{H}$ & 19. 099 & $1 U$ & - & 17791.51 & ...... & $-10-10$ & C & 4350.328 & (15) & II & 22980.334 & +0.003 & $6 p$ & ${ }^{3} \mathrm{P}_{1}^{0}-6 p^{2}{ }^{3} \mathrm{P}_{2}$ \\
\hline G & 5618.7 & $1 u$ & $\ldots$ & 17792.77 & & & $\mathrm{C}$ & 4332.914 & (3) & $10 n$ & 23072.692 & 0.000 & $6 p$ & ${ }^{3} \mathrm{P}_{1} \mathrm{i}-7 d{ }^{3} \mathrm{D}_{1}$ \\
\hline $\mathrm{H}$ & 5593. 297 & 3 & 3 III & 17873.58 & +0.02 & $6 p \quad{ }^{1} \mathrm{P}_{1}^{\circ}-6 d^{\prime} \quad{ }^{3} \mathrm{D}_{1}$ & $\mathrm{H}$ & 4325.152 & 3 & 10 & 23114.07 & -0.06 & $6 p$ & ${ }^{3} \mathrm{P}_{2}^{0}-6 d^{\prime}{ }^{3} \mathrm{D}_{3}$ \\
\hline $\mathrm{G}$ & $\begin{array}{l}5546 . \\
5535\end{array}$ & $2 u$ & (n) & $\begin{array}{l}18025.68 \\
18059.02\end{array}$ & +004 & & $\mathrm{C}$ & 4323. 620 & & & 23122. 286 & & & \\
\hline $\begin{array}{l}\mathrm{F} \\
\mathrm{C}\end{array}$ & $\begin{array}{l}5535.86 \\
5535.484\end{array}$ & $(100 R)$ & $1000 R \mathrm{I}$ & $\begin{array}{l}18059.02 \\
18060.264\end{array}$ & $\begin{array}{r}+0.04 \\
0.000\end{array}$ & $\begin{array}{lll}6 p & { }^{3} \mathrm{P}_{\mathrm{i}}-6 d & { }^{3} \mathrm{D}_{1} \\
6 s^{2} & { }_{1} \mathrm{~S}_{0}-6 p & 1 \mathrm{P}^{\circ}\end{array}$ & $\mathrm{C}$ & $\begin{array}{l}\text { 4323. } 001 \\
4305.15\end{array}$ & $(3 v)$ & $20 n$ III & $\begin{array}{l}23125.597 \\
23221.46\end{array}$ & $\begin{array}{l}+0.002 \\
+0.16\end{array}$ & $\begin{array}{l}6 p \\
5 d\end{array}$ & $\begin{array}{ll}{ }^{3} \mathrm{P}_{1}-7 d & { }^{3} \mathrm{D}_{2} \\
{ }^{1} \mathrm{D}_{2}-4 f & { }^{3 \mathrm{~F}_{3}}\end{array}$ \\
\hline $\mathrm{C}$ & 5519.047 & $(50)$ & 200 II & 18114. 052 & -0.001 & $\begin{array}{lll}6 s^{2} & \mathrm{~S}_{0}-6 p & \mathrm{P}_{1} \\
6 p & 3 \mathrm{P}_{1}^{\circ}-6 d & 3 \mathrm{D}_{2}\end{array}$ & C & $\begin{array}{l}4305.15 \\
4291.158\end{array}$ & $\begin{array}{l}1 \\
(5)\end{array}$ & 12 & $\begin{array}{l}23221,46 \\
23297.201\end{array}$ & $\begin{array}{l}+0.10 \\
-0.008\end{array}$ & $\begin{array}{l}5 a \\
6 p\end{array}$ & $3 \mathrm{P}_{1}^{0}-6 d^{\prime} 3 \mathrm{D}_{1}$ \\
\hline H & 5473.689 & $2 u$ & 3 III $A$ & 18264.14 & & & $\mathrm{I}$ & 4284.90 & 1 & & 23331. 20 & -0.36 & $5 d$ & ${ }^{3} \mathrm{D}_{2}-7 p{ }^{1} \mathrm{Pi}_{\mathrm{i}}^{1}$ \\
\hline$\overline{\mathrm{H}}$ & 5437. 393 & $2 u$ & $4 \operatorname{III} A$ & 18386.06 & -0.26 & $6 p{ }^{1} \mathrm{P}_{1}^{0}-6 d^{\prime}{ }^{3} \mathrm{~S}_{1}$ & $\mathrm{C}$ & 4283. & $(20)$ & 100 & 23341.041 & 0.000 & $5 d$ & ${ }^{1} \mathrm{D}_{2}-4 f \quad 1 \mathrm{~F}_{3}^{\circ}$ \\
\hline $\mathrm{C}$ & 5424.551 & $(50 r)$ & 100 II & 18429.597 & +0.024 & $6 p{ }^{3} \mathrm{P}_{0}^{0}-6 d \quad{ }^{3} \mathrm{D}_{1}$ & I & 4278.83 & $0+$ & & 23364.50 & & & \\
\hline $\mathrm{H}$ & 5416.344 & $1 U$ & 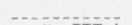 & 18457.51 & ...... & - & C & 4264,418 & (2) & $15 n \operatorname{III} A$ & 23443. 286 & -0.001 & $6 p$ & ${ }^{3} \mathrm{P}_{0}^{\circ}-7 d{ }^{3} \mathrm{D}_{1}$ \\
\hline $\mathrm{H}$ & 5404. 920 & $1 u$ & $5 n \operatorname{III} A$ & 18496.52 & ..... & - & I & 4252.4 & 1 & & & & & \\
\hline I & 5393. 25 & $1 h R$ & -.......... & 18536.54 & $\ldots \ldots$ & - & $\mathrm{C}$ & 4242.606 & (4) & 10 & 23563.807 & 0.000 & $6 p$ & ${ }^{3} \mathrm{P}_{1}^{\circ}-6 d^{\prime}{ }^{3} \mathrm{D}_{2}$ \\
\hline $\mathrm{G}$ & 5389.6 & $1 u$ & $\ldots$ & 18549.10 & -..... & - & C & 4239.557 & (2) & $10 n$ & 23580 & 0. & $6 p$ & ${ }^{3} \mathrm{P}_{2}^{0}-9 s \quad{ }^{3} \mathrm{~S}_{1}$ \\
\hline $\mathrm{G}$ & 5381. ( & $1 u$ & $\ldots$ & 18578.75 & $\ldots$ & 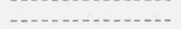 & $\mathrm{C}$ & 4223.962 & (5) & 12 III & 23667.813 & +0.009 & $6 p$ & ${ }^{3} \mathrm{P}_{0}^{0}-6 d^{\prime}{ }^{3} \mathrm{D}_{1}$ \\
\hline $\mathrm{K}$ & 5378.85 & (1) & .... & 18586.17 & ... & (........ & $\mathrm{H}$ & 4179. & $2 r$ & $8 n \operatorname{III} A$ & 23920.30 & +0.0 & $6 p$ & ${ }^{3} \mathrm{P}_{2}^{0}-7 d \quad{ }^{1} \mathrm{D}_{2}$ \\
\hline $\mathrm{H}$ & 5365.383 & $1 u$ & - n & 18632.82 & & $\ldots$ & $\mathrm{E}$ & 4134. 38 & 1 & ters & & & $6 p$ & ${ }^{3} \mathrm{P}_{0}^{0}-6 d^{\prime}{ }^{3} \mathrm{~S}_{1}$ \\
\hline $\mathrm{H}$ & 5349. & $1 U$ & & 18687.72 & ....... & - & $\mathrm{C}$ & 4132.427 & (5) & 20 & 24192.054 & -0.003 & $6 s^{2}$ & ${ }^{1} \mathrm{~S}_{0}-6 p^{\prime}{ }^{2} \mathrm{Di}$ \\
\hline $\mathrm{H}$ & 5308.952 & $1 U$ & 4 IV? & 18830.87 & & & $\mathrm{E}$ & 4110.23 & 2 & 2 III & 24322.67 & +0.0 & $6 p$ & ${ }^{3} \mathrm{P}_{2}^{\circ}-6 d^{\prime}{ }^{1} \mathrm{D}_{2}$ \\
\hline $\mathrm{H}$ & 5305.758 & $2 u$ & 4 IV? & 18842. 20 & -0.09 & $6 p{ }^{1} \mathrm{P}_{1}^{0}-6 d^{\prime}{ }^{1} \mathrm{P}_{1}$ & $\mathrm{H}$ & 4087.371 & $1 U$ & $8 n \operatorname{III} A$ & 24458. 70 & & $6 p$ & ${ }^{3} \mathrm{P}_{1}^{\circ}-9 s \quad{ }^{3} \mathrm{~S}_{1}$ \\
\hline $\mathrm{H}$ & 5302. 808 & $3 u$ & 6 IV? & 18852.69 & $\ldots \ldots$ & - & & 4007.011 & 10 & & & -0.7 & $6 p$ & ${ }^{3} \mathrm{P}_{2}^{\circ}-8 d \quad{ }^{3} \mathrm{D}_{2}$ \\
\hline $\mathrm{H}$ & 5294. 130 & $2 u$ & (n) & 18883.59 & ....... & 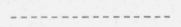 & $\mathrm{H}$ & 4085. 322 & $1 U$ & & 24470.97 & & & \\
\hline $\mathrm{H}$ & 5290.945 & $1 U$ & $\ldots$ & 18894. 96 & -...... & 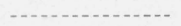 & $\mathrm{C}$ & 4084 & (1u) & $30 N$ III & 24473. 747 & 0.0 & $6 p$ & ${ }^{3} \mathrm{P}_{2}^{\circ}-8 d \quad{ }^{3} \mathrm{D}_{3}$ \\
\hline $\mathrm{H}$ & 5279. 619 & $1 U$ & $\ldots$ & 18935.49 & -... & - & G & & $1 u$ & $6 n$ III & 24497 & & $5 d$ & ${ }^{1} \mathrm{D}_{2}-8 p{ }^{1} \mathrm{Pi}$ \\
\hline $\mathrm{H}$ & 5277.625 & $1 U$ & 3 IV? & 18942.64 & & & $\mathrm{E}$ & 40 & $1 \mathrm{~Pb}$ ? & -..... & 24636 & +0.2 & $6 p$ & ${ }^{3} \mathrm{P}_{0}^{0}-6 d^{\prime}{ }^{1} \mathrm{P}_{1}$ \\
\hline $\mathrm{H}$ & 5267.033 & $4 u$ & 20 IV? & 18980.74 & 0.00 & $6 p{ }^{1} \mathrm{P}_{1}-8 s{ }^{1} \mathrm{~S}_{0}$ & $\mathrm{E}$ & 797 & 0 & - & & -0.0 & $6 p$ & ${ }^{3} \mathrm{P}_{2}^{0}-6 d^{\prime}{ }^{3} \mathrm{P}_{2}$ \\
\hline $\mathrm{H}$ & 5265.566 & $1 u$ & -.............. & 18986. 03 & & & $\mathrm{E}$ & & $0 h$ & & 18 & -0.16 & $6 p$ & ${ }^{3} \mathrm{P}_{1}^{0}-7 d \quad{ }^{1} \mathrm{D}_{2}$ \\
\hline $\mathrm{H}$ & 5253.807 & $1 U$ & & 19028.52 & -0.06 & $6 p{ }^{1} \mathrm{P}_{1}^{0}-6 d^{\prime}{ }^{8} \mathrm{~F}_{2}$ & $\mathrm{G}$ & 4026.40 & $1 r$ & IV? & & -0.4 & $6 p$ & ${ }^{3} \mathrm{P}_{0}^{0}-9 s \quad{ }^{3} \mathrm{~S}_{1}$ \\
\hline $\mathrm{E}$ & 5177.322 & $(2 u)$ & 2 IV? & 19309.63 & & - & G & 39 & 1 & III & 06 & -0.19 & $5 d$ & ${ }^{3} \mathrm{D}_{3}-4 f \quad{ }^{3} \mathrm{~F}_{2}^{2}$ \\
\hline $\mathrm{H}$ & 5175.619 & $3 u$ & 5 IV? & 19315.98 & & & $\mathrm{C}$ & 3995. 656 & (5) & 30 & 25020.131 & -0.001 & $5 d$ & ${ }^{3} \mathrm{D}_{3}-4 f \quad{ }^{3} \mathrm{~F}_{3}^{0}$ \\
\hline $\mathrm{E}$ & 5169.490 & 1 & & 19338.88 & +0.14 & $5 \bar{d}{ }^{3} \mathrm{D}_{2}-6 p^{\prime}{ }^{1} \mathrm{P}_{i}$ & C & 3993. 401 & (100) & 80 & 25034. 258 & 0.000 & $5 d$ & ${ }^{3} \mathrm{D}_{3}-4 f \quad 3 \mathrm{~F}_{4}^{\circ}$ \\
\hline $\mathrm{C}$ & 5159.939 & (5) & $20 n$ IV? & 19374. 692 & 0.000 & $6 p \quad{ }^{1} \mathrm{P}_{1}^{0}-7 d \quad{ }^{1} \mathrm{D}_{2}$ & $\mathrm{E}$ & 3992.255 & $3 u$ & & 25041.42 & -0.02 & $6 p$ & ${ }^{3} \mathrm{P}_{2}^{\circ}-8 d{ }^{1} \mathrm{D}_{2}$ \\
\hline G & 5093. 9 & 1 & - & 19625.87 & & & C & & (1) & III & 25100. 399 & +0.011 & $5 d$ & ${ }^{1} \mathrm{D}_{2}-7 p^{\prime}{ }^{3} \mathrm{D}_{1}$ \\
\hline $\mathrm{H}$ & 5054.975 & $2 u$ & 5 IV? & 19776.98 & -0.16 & $6 p \quad{ }^{1} \mathrm{P}_{1}^{\circ}-6 d^{\prime} \quad{ }^{1} \mathrm{D}_{2}$ & $\mathrm{~K}$ & 3980.42 & $2 n$ & & 25115.87 & -0.01 & $5 d$ & ${ }^{1} \mathrm{D}_{2}-7 p^{\prime}{ }^{3} \mathrm{~F}_{3}^{0}$ \\
\hline G & 4995.72 & 1 & -- & 20011.55 & -0.12 & $6 p{ }^{3} \mathrm{P}_{2}^{\circ}-7 s^{\prime} \quad{ }^{3} \mathrm{D}_{3}$ & $\mathrm{C}$ & 3975.365 & $(1 u)^{\prime}$ & & 25147.834 & -0.34 & $6 p$ & ${ }^{3} \mathrm{P}_{2}^{0}-108 \quad{ }^{2} \mathrm{~S}_{1}$ \\
\hline $\mathrm{H}$ & 4947.350 & $3 u$ & 8 III & 20207.20 & & $6 p{ }^{1}{ }^{1} \mathrm{P}_{1}^{\circ}-9 s{ }^{1}{ }^{1} \mathrm{~S}_{0}$ & $\mathrm{C}$ & 3947.507 & $(1 u)$ & 51 & 25325. 303 & 0.000 & $6 p$ & ${ }^{3} \mathrm{P}_{\mathrm{i}}^{\mathrm{i}}-8 d{ }^{3}{ }^{2} \mathrm{D}_{1}$ \\
\hline $\mathrm{E}$ & 4929. 355 & 1 & & 20280.97 & $\begin{array}{l}-0.21 \\
-0.13\end{array}$ & $\begin{array}{lll}6 p & 1 \mathrm{P}_{1}^{1}-6 d^{\prime} & { }^{3} \mathrm{P}_{2} \\
6 p & { }^{3} \mathrm{P}_{2}^{0}-7 s^{\prime} & { }^{1} \mathrm{D}_{2}\end{array}$ & C & $\begin{array}{l}39 \\
39\end{array}$ & (8) & $\begin{array}{l}10 N \text { III } \\
20 \text { III }\end{array}$ & $\begin{array}{l}25337.575 \\
25387.279\end{array}$ & $\begin{array}{r}0.000 \\
-0.005\end{array}$ & $\begin{array}{l}6 p \\
5 d\end{array}$ & $\begin{array}{ll}{ }^{3} \mathrm{P}_{\mathrm{i}}-8 d & { }^{3} \mathrm{D}_{2} \\
{ }^{3} \mathrm{D}_{2}-4 f & 3 \mathrm{~F}_{2}^{0}\end{array}$ \\
\hline$\vec{H}$ & & $4 r$ & 15 III & & -0.20 & $6 p \quad{ }^{3} \mathrm{P}_{2}^{0}-8 s \quad{ }^{3} \mathrm{~S}_{1}$ & C & & (50) & 50 & 25401. 165 & & $5 d$ & ${ }^{3} \mathrm{D}_{2}-4 f \quad 3 \mathrm{~F}_{3}^{0}$ \\
\hline$\vec{H}$ & 4877.650 & $3 u$ & 30 NIII & 20495.95 & +0.03 & $6 p \quad{ }^{1} \mathrm{P}_{1}^{0}-8 d \quad{ }^{1} \mathrm{D}_{2}$ & $\mathrm{~K}$ & 3917.26 & 8 & III & 25520.82 & -0.08 & $5 d$ & ${ }^{3} \mathrm{D}_{2}-4 f \quad{ }^{1} \mathrm{~F}_{3}^{0}$ \\
\hline G & 4807.6 & $1 u$ & $2 N I I I$ & 20794. 59 & H..... & $\begin{array}{l}-2-1 \\
\end{array}$ & $\mathrm{C}$ & 3909.910 & $(50 r)$ & 40 & 25568.821 & +0.004 & $5 d$ & ${ }^{3} \mathrm{D}_{1}-4 f \quad{ }^{3} \mathrm{~F}_{2}^{\circ}$ \\
\hline G & 4739.3 & $1 u$ & 5 NIII & 21094. 26 & & & $\mathrm{E}$ & 3905.986 & $2 h$ & III & 25594.48 & -0.15 & $5 d$ & ${ }^{1} \mathrm{D}_{2}-7 p^{\prime}{ }^{3} \mathrm{P}_{\mathrm{i}}^{\circ}$ \\
\hline G & 4731.8 & $1 u$ & 5 NIII? & 21127.70 & -..... & & G & 3900.41 & 1 & 2 III & 25631. 07 & +0.02 & $6 p$ & ${ }^{3} \mathrm{P}_{\mathrm{i}}-6 d^{\prime}{ }^{3} \mathrm{P}_{2}$ \\
\hline $\mathrm{C}$ & 4726.436 & (10) & 40 II & 21151.692 & -0.002 & $5 d{ }^{1} \mathrm{D}_{2}-7 p{ }^{1} \mathrm{P}_{\mathrm{i}}^{0}$ & I & 3898.58 & 0 & & 25643.10 & -0.05 & $6 p$ & ${ }^{3} \mathrm{P}_{2}^{0}-9 d \quad{ }^{3} \mathrm{D}_{2}$ \\
\hline
\end{tabular}


TABle 2-Continued

\begin{tabular}{|c|c|c|c|c|c|c|c|c|c|c|c|c|c|}
\hline \multirow{2}{*}{$\begin{array}{l}\text { Lit. } \\
\text { Ref. }\end{array}$} & \multirow{2}{*}{ I. A. } & \multicolumn{2}{|c|}{ Intensity } & \multirow{2}{*}{ K } & \multirow{2}{*}{$\begin{array}{l}\Delta \mathrm{K} \\
\mathrm{O}-\mathrm{c}\end{array}$} & \multirow{2}{*}{ Desig. } & \multirow{2}{*}{$\begin{array}{l}\text { Lit. } \\
\text { Ref. }\end{array}$} & \multirow{2}{*}{ I. A. } & \multicolumn{2}{|c|}{ Intensity } & \multirow{2}{*}{ K } & \multirow{2}{*}{$\begin{array}{l}\Delta \mathrm{K} \\
0-\mathrm{c}\end{array}$} & \multirow{2}{*}{ Desig. } \\
\hline & & Ref. & $\begin{array}{l}\text { King } \\
\text { Are T. C. }\end{array}$ & & & & & & Ref. & $\begin{array}{c}\text { King } \\
\text { Are T. C. }\end{array}$ & & & \\
\hline $\mathrm{C}$ & 3894,345 & $1 h$ & & 25671.013\{ & $\begin{array}{r}+3.08 \\
0.00\end{array}$ & $5 d{ }^{1}{ }^{1} \mathrm{D}_{2}-7 p^{\prime}{ }^{3} \mathrm{D}_{2}^{\circ}$ & $\mathrm{F}$ & 3426.453 & & $4 n$ III? & 29176.33 & & \\
\hline $\mathrm{H}$ & 3892.653 & $2 u$ & $20 \quad$ III & 25682.14 & $\begin{array}{r}0.00 \\
+0.02\end{array}$ & $\begin{array}{ll}6 p & { }^{3} \mathrm{P}_{2}^{0}-9 d \\
5 d & { }^{1} \mathrm{D}_{2}-7 \mathrm{D}_{3} \\
p^{\prime} & { }^{1} \mathrm{D}_{2}^{\circ}\end{array}$ & $\mathrm{F}$ & 3421.476 & & {$[30 n]$ III } & 29218.77 & $\begin{array}{r}0.00 \\
-0.19\end{array}$ & $\begin{array}{cll}5 d & 1 \mathrm{D}_{2}-9 f & 1 \mathrm{~F}_{0}^{\circ} \\
(5 d & 1 \mathrm{D}_{2}-9 f & \left.3 \mathrm{~F}_{2}^{\circ}\right)\end{array}$ \\
\hline I & $\begin{array}{l}3890.57 \\
3889.326\end{array}$ & 0 & $\ldots$ & 25695.89 & -0.01 & $6 p{ }^{3} \mathrm{P}_{0}^{0}-8 d{ }^{3} \mathrm{D}_{1}$ & $\mathrm{~F}$ & 3421.008 & & [25] III & 29222.77 & +0.90 & $5 d{ }^{3} \mathrm{D}_{3}-6 f{ }^{3} \mathrm{~F}_{3}^{0}$ \\
\hline $\mathrm{E}$ & $\begin{array}{l}3889.326 \\
3881.319\end{array}$ & (8) & $20 \quad$ II & 25704.140 & 0.000 & $6 s^{2}{ }^{1} \mathrm{~S}_{0}-6 p^{\prime}{ }^{3} \mathrm{P}_{1}^{\circ}$ & $\mathrm{C}$ & 3420.316 & $(8 r)$ & {$[70 R]$ III } & 29228.714 & 0.000 & $5 d{ }^{3} \mathrm{D}_{3}-6 f{ }^{3} \mathbf{F}_{4}^{\circ}$ \\
\hline & 3881.319 & & - & 25757.14 & $\begin{array}{l}-0.06 \\
-0.08\end{array}$ & $6 p{ }^{3} \mathrm{P}_{0}^{\circ}-6 d^{\prime}{ }^{3} \mathrm{P}_{1}$ & $\mathrm{~F}$ & 3413.835 & $\ldots$ & $3 n$ III & 29284.17 & -0.16 & $5 d{ }^{3} \mathrm{D}_{2}-7 p^{\prime}{ }^{1} \mathrm{P}_{1}^{\circ}$ \\
\hline $\mathrm{H}$ & 3861.905 & $2 u$ & $15 \quad$ III & 25886.62 & $\begin{array}{l}-0.08 \\
-0.17\end{array}$ & 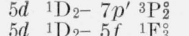 & $\mathrm{F}$ & 3377. 391 & $(5 r)$ & [20] III & 29600.15 & -0.07 & $5 d \quad 3 \mathrm{D}_{2}-6 f \quad 3 \mathrm{~F}_{2}^{\circ}$ \\
\hline I & 3841.15 & $1 h$ & ........... & 26026.49 & +0.20 & $6 p \quad 3 \mathrm{P}_{1}^{0}-10 s \quad 3 \mathrm{~S}_{1}$ & $\stackrel{\mathrm{C}}{\mathrm{C}}$ & $\begin{array}{l}3377.081 \\
3356.800\end{array}$ & $\begin{array}{l}(5 r) \\
(4 r)\end{array}$ & $\begin{array}{l}{[60 R] \text { III }} \\
80 N \text { III }\end{array}$ & $\begin{array}{l}29602.900 \\
29781.750\end{array}$ & $\begin{array}{l}0.000 \\
0.000\end{array}$ & $\begin{array}{lll}5 d & 3 \mathrm{D}_{2}-6 f & 3 \mathrm{~F}_{3}^{0} \\
5 d & 3 \mathrm{D}_{1}-6 f & 3 \mathrm{~F}_{2}^{0}\end{array}$ \\
\hline I & 3828.93 & - & & 26109.55 & -0.40 & $6 p \quad{ }^{3} \mathrm{P}_{2}^{0}-11 s \quad{ }^{3} \mathrm{~S}_{1}$ & $\mathrm{~F}$ & 3323.058 & (17) & [10] III & 30084.11 & $\begin{array}{l}0.000 \\
+0.04\end{array}$ & 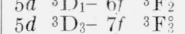 \\
\hline$\underset{\mathrm{J}}{\mathrm{H}}$ & $\begin{array}{l}3794.771 \\
3790.27\end{array}$ & $\begin{array}{l}5 v \\
0\end{array}$ & 15 & $\begin{array}{l}26344.57 \\
26375.85\end{array}$ & 0.00 & $5 d{ }^{1} \mathrm{D}_{2}-7 p^{\prime}{ }^{1} \mathrm{~F}_{3}^{\circ}$ & $\mathrm{F}$ & 3322. 797 & 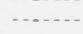 & [50r] III & 30086.47 & 0.00 & $5 d \quad{ }^{3} \mathrm{D}_{3}-7 f{ }^{3} \mathrm{~F}_{4}^{\circ}$ \\
\hline I & 3789.72 & $-n l$ & & 26379.68\{ & -0.11 & $6 p{ }^{3} \mathrm{P}_{2}^{\circ}-10 d \quad{ }^{3} \mathrm{D}_{2}$ & G & $\begin{array}{l}3315.753 \\
3298.2\end{array}$ & $1 r$ & $8 n$ III & $\begin{array}{l}30150.39 \\
30310.84\end{array}$ & -..... & - \\
\hline I & 3788.18 & - & & 26390.41 & $\begin{array}{r}+0.56 \\
0.00\end{array}$ & 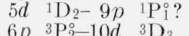 & F & 3281.772 & & [15] III & 30462.56 & +0.02 & $5 d{ }^{3} \mathrm{D}_{2}-7 f \quad{ }^{3} \mathrm{~F}_{2}^{\circ}$ \\
\hline I & 3787.23 & - & & 26397. 03 & $\begin{array}{r}0.00 \\
+0.14\end{array}$ & $\begin{array}{lll}6 p & 3 \mathrm{P}_{2}^{\circ}-10 d & { }^{3} \mathrm{D}_{3} \\
6 p & { }^{3} \mathrm{P}_{0}^{\circ}-10 s & { }^{3} \mathrm{~S}_{1}\end{array}$ & $\mathrm{~F}$ & $\begin{array}{l}3281.503 \\
3272.405\end{array}$ & & [40r] III & 30465.06 & -0.04 & $5 d{ }^{3} \mathrm{D}_{2}-7 f \quad{ }^{3} \mathrm{~F}_{3}^{\circ}$ \\
\hline I & 3771.93 & - & & 26504.10 & +0.02 & 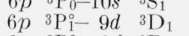 & $\mathrm{F}$ & $\begin{array}{l}3272.405 \\
3270.115\end{array}$ & & $\begin{array}{ll}2 n & \text { III } \\
4 n & \text { III }\end{array}$ & $\begin{array}{l}30549.76 \\
30571.15\end{array}$ & -0.03 & $5 d{ }^{3} \mathrm{D}_{2}-39765^{\circ}$ \\
\hline I & $\begin{array}{l}3769.48 \\
3767.4\end{array}$ & $\overline{0} H$ & $\ldots$ & 26521. 32 & +0.05 & $6 p{ }^{3} \mathrm{P}_{1}^{\circ}-9 d \quad{ }^{3} \mathrm{D}_{2}$ & $\mathrm{~F}$ & 3262.336 & & {$\left[\begin{array}{lll}60 & P\end{array}\right]$ III } & 30644.05 & -0.03 & $5 d{ }^{3} \mathrm{D}_{1}-7 f{ }^{3} \mathrm{~F}_{2}^{0}$ \\
\hline I & & $\underline{0 H}$ & & 26535.96 & & & $\mathrm{~F}$ & 3261.961 & ..... & {$[50 r]$ III } & 30647.57 & +0.10 & $5 d{ }^{3} \mathrm{D}_{3}-8 f{ }^{3} \mathrm{~F}_{4}^{0}$ \\
\hline 1 & $\begin{array}{l}3721.17 \\
3720.85\end{array}$ & E & $\cdots$ & 26865. 63 & +0.05 & $6 p \quad{ }^{3} \mathrm{P}_{2}^{\circ}-11 d \quad 3 \mathrm{D}_{2}$ & $\mathrm{~F}$ & 3253.067 & ..... & $5 n$ III & 30731.36 & +0.03 & $5 d{ }^{3} \mathrm{D}_{1}-39765^{\circ}$ \\
\hline I & $\begin{array}{l}3720.85 \\
3719.92\end{array}$ & - & & 26867.94 & 0.00 & $\begin{array}{llll}6 p & 3 \mathrm{P}_{2}^{\circ}-11 d & 3 \mathrm{D}_{3}\end{array}$ & $J$ & 3244.20 & & & 30815.35 & -0.21 & $6 s^{2} \quad{ }^{1} \mathrm{~S}_{0}-7 p{ }^{3} \mathrm{P}_{1}^{\circ}$ \\
\hline I & 3704.23 & - & & $\begin{array}{l}26874.65 \\
26988.48\end{array}$ & -0.03 & $\begin{array}{llll}6 p & 3 \mathrm{P}_{0}^{\circ}-9 d & 3 \mathrm{D}_{1}\end{array}$ & $F$ & 3222.441 & $-\ldots \ldots$ & [8] & 31023.42 & +0.03 & $5 d \quad{ }^{3} \mathrm{D}_{2}-8 f \quad{ }^{3} \mathrm{~F}_{2}^{\circ}$ \\
\hline $\mathrm{H}$ & 3701.716 & $2 u$ & III & $\begin{array}{l}26988.48 \\
27006.81\end{array}$ & & $6 p{ }^{3} \mathrm{P}_{1}^{\circ}-11 s \quad{ }^{3} \mathrm{~S}_{1}$ & $\mathrm{~F}$ & 3222.188 & -- & [40] & 31025.85 & 0.00 & $5 d \quad{ }^{3} \mathrm{D}_{2}-8 f{ }^{3} \mathrm{~F}_{3}^{\circ}$ \\
\hline $\mathrm{K}$ & 3699.94 & 2 & & 27019. 78 & +0.44 & $5 d{ }^{3} \mathrm{D}_{2}-7 p^{\prime}{ }^{3} \mathrm{~F}_{2}^{\circ}$ & $\mathrm{F}$ & $\begin{array}{l}3221.630 \\
3203.700\end{array}$ & & $\begin{array}{l}{[30] \mathrm{III}} \\
25 \mathrm{~N} \text { III }\end{array}$ & 31031. 23 & 0.00 & $5 d{ }^{3} \mathrm{D}_{3}-9 f{ }^{3} \mathrm{~F}_{4}^{0}$ \\
\hline $\mathrm{H}$ & 3688.473 & $3 r$ & $20 N$ III & 27103.78 & -0.69 & $5 d \quad{ }^{1} \mathrm{D}_{2}-7 p^{\prime}{ }^{1} \mathrm{P}_{1}$ & $\mathrm{~F}$ & 3193.967 & & $\begin{array}{l}25 N \\
{[5] \text { III }}\end{array}$ & $\begin{array}{l}31204.89 \\
31299.98\end{array}$ & -0.04 & $5 d{ }^{3} \mathrm{D}_{1}-8 f{ }^{3} \mathrm{~F}_{2}^{0}$ \\
\hline $\mathrm{C}$ & 3675.310 & (1) & 2 III & 27200.873 & 0.000 & $5 d{ }^{3} \mathrm{D}_{1}-7 p^{\prime}{ }^{3} \mathrm{~F}_{2}^{\circ}$ & $\mathrm{F}$ & 3193.912 & & [10] & 31300.52 & 0.00 & $5 d{ }^{3} \mathrm{D}_{3}-10 f \quad{ }^{3} \mathrm{~F}_{4}^{\circ}$ \\
\hline I & 3667.93 & 一 & $\ldots$ & 27255.57 & +0.01 & $6 p \quad{ }^{3} \mathrm{P}_{\mathrm{i}}^{\circ}-10 d \quad{ }^{3} \mathrm{D}_{1}$ & $\mathrm{~F}$ & 3183.96 & & {$[15]$} & 31398.35 & -0.29 & $5 d{ }^{3} \mathrm{D}_{2}-9 f \quad{ }^{3} \mathrm{~F}_{2}^{0}$ \\
\hline 1 & $\begin{array}{l}3667.60 \\
3664.598\end{array}$ & - & & 27258.02 & +0.11 & $6 p \quad{ }^{3} \mathrm{P}_{1} \mathrm{i}-10 d \quad{ }^{3} \mathrm{D}_{2}$ & $\mathrm{~F}$ & 3183.156 & & [30] III & 31406.28 & 0.00 & $5 d \quad{ }^{3} \mathrm{D}_{2}-9 f \quad{ }^{3} \mathrm{~F}_{3}^{\circ}$ \\
\hline$\stackrel{\mathrm{H}}{\mathrm{C}}$ & $\begin{array}{l}3664.598 \\
3662.535\end{array}$ & $\begin{array}{l}1 u \\
(3)\end{array}$ & III & 27280.35 & +0.10 & $5 d{ }^{3} \mathrm{D}_{2}-7 p^{\prime}{ }^{3} \mathrm{D}_{1}^{0}$ & $\mathrm{~F}$ & 3173.69 & $-\ldots$ & {$[15 n]$ III } & 31499.95 & 0.00 & $5 d \quad{ }^{3} \mathrm{D}_{3}-11 f \quad{ }^{3} \mathrm{~F}_{4}^{\circ}$ \\
\hline $\mathrm{C}$ & $\begin{array}{l}3002.535 \\
3640.391\end{array}$ & $\begin{array}{l}\text { (3) } \\
(3)\end{array}$ & 15 & 27295. 748 & 0.000 & $5 d{ }^{3} \mathrm{D}_{2}-7 p^{\prime}{ }^{3} \mathrm{~F}_{3}^{\circ}$ & $\mathrm{F}$ & 3165.598 & & [25] III & 31580.47 & +0.29 & $5 d{ }^{3} \mathrm{D}_{1}-9 f \quad{ }^{3} \mathrm{~F}_{2}^{\circ}$ \\
\hline $\mathrm{F}$ & 3639. 715 & (3) & & 27461.780 & -0.005 & $5 d{ }^{3} \mathrm{D}_{1}-7 p^{\prime}{ }^{3} \mathrm{D}_{1}^{\circ}$ & $\mathrm{F}$ & 3158.54 & $-\ldots$ & {$[12 N] \mathrm{III}$} & 31651.03 & 0.00 & $5 d \quad{ }^{3} \mathrm{D}_{3}-12 f \quad{ }^{3} \mathrm{~F}_{4}^{\circ}$ \\
\hline $\mathrm{F}$ & 3636.832 & & $\stackrel{2}{2}$ III & $\begin{array}{l}27466.85 \\
27488.62\end{array}$ & $\begin{array}{r}+0.09 \\
0.00\end{array}$ & $\begin{array}{l}5 d{ }^{3} \mathrm{D}_{3}-7 p^{\prime}{ }^{3} \mathrm{D}_{2}^{\circ} \\
5 d\end{array}$ & $\mathrm{~F}$ & 3158.046 & & [12] III & 31655.99 & & \\
\hline $\mathrm{C}$ & 3630.640 & $(10)$ & 40 III & $\begin{array}{l}27400.02 \\
27535.533\end{array}$ & 0.000 & $\begin{array}{l}5 d{ }^{1} \mathrm{D}_{2}-6 f \\
5 d \\
5{ }^{3} \mathrm{D}_{3}-7 p^{\prime}{ }^{3} \mathrm{~F}_{3}^{3}\end{array}$ & $\frac{\mathrm{F}}{\mathrm{F}}$ & $\begin{array}{l}3155.673 \\
3155.336\end{array}$ & & {$[10]$} & $\begin{array}{l}31679.79 \\
31682\end{array}$ & +0.04 & $\begin{array}{lll}5 d & { }^{3} \mathrm{D}_{2}-10 f & { }^{3} \mathrm{~F}_{2}^{\circ} \\
5 d & & \end{array}$ \\
\hline $\mathrm{C}$ & 3610.957 & - n..... & $15 n$ III & $27685.624\}$ & 0.000 & $5 d \quad{ }^{3} \mathrm{D}_{3}-5 f \quad{ }^{1} \mathrm{~F}_{3}^{0}$ & $\mathrm{I}$ & 3146.90 & & {$[10]$} & $\begin{array}{l}31683.17 \\
31768.10\end{array}$ & $\begin{array}{l}0.00 \\
0.00\end{array}$ & $\begin{array}{lll}5 d & 3 \mathrm{D}_{2}-10 f & { }^{3} \mathrm{~F}_{3}^{3} \\
5 d & { }^{3} \mathrm{D}_{3}-13 f & { }_{3}^{3} \mathrm{~F}_{4}^{\circ}\end{array}$ \\
\hline I & 3603.40 & - & & & +0.09 & $5 d{ }^{3} \mathrm{D}_{3}-7 p^{\prime}{ }^{3} \mathrm{P}_{2}^{0}$ & I & 3137.80 & & & 31860.23 & 0.00 & $5 d{ }^{3} \mathrm{D}_{3}-14 f{ }^{3} \mathrm{~F}^{\circ}$ \\
\hline$\stackrel{1}{\mathrm{C}}$ & 3599. 402 & (3) & III & 27743.65 & -0.05 & $6 p{ }^{3} \mathrm{P}_{\mathrm{i}} \mathrm{i}-11 \mathrm{~d},{ }^{3} \mathrm{D}_{2}$ & $\mathrm{~F}$ & 3137.700 & & [10] III & 31861.25 & -0.04 & $5 d{ }^{3} \mathrm{D}_{1}-10 f \quad{ }^{3} \mathrm{~F}_{2}$ \\
\hline I & 3596.33 & $(1 u)$ & 15 & $\begin{array}{l}27774.499 \\
27798.19\end{array}$ & $\begin{array}{l}+0.001 \\
-0.18\end{array}$ & 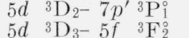 & $\mathrm{G}$ & $\begin{array}{l}3135.72 \\
3132602\end{array}$ & & {$[8 n]$ III } & 31881.36 & 0.00 & $5 d{ }^{3} \mathrm{D}_{2}-11 f \quad{ }^{3} \mathrm{~F}_{3}^{\circ}$ \\
\hline $\mathrm{C}$ & 3593. 204 & (1) & $15 n$ III & 27822.406 & -0.010 & 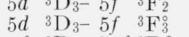 & I & 3130.6 & & {$\left[\begin{array}{ll}{[8]} & 111 \\
-\end{array}\right.$} & $\begin{array}{l}31913.10 \\
31933.50\end{array}$ & 0.00 & $5 d^{3} \mathrm{D}-15 f^{3} \mathrm{~F}^{\circ}$ \\
\hline $\mathrm{F}$ & $\begin{array}{l}3589.950 \\
3588.099\end{array}$ & $\ldots \ldots$ & 3 IIIA & 27847.59 & -0.20 & $5 d \quad{ }^{3} \mathrm{D}_{2}-7 p^{\prime}{ }^{3} \mathrm{D}_{2}^{\circ}$ & I & 3121.02 & - & & 32031.52 & 0.00 & $5 d \quad{ }^{3} \mathrm{D}_{2}-12 f \quad{ }^{3} \mathrm{~F}_{3}^{\circ}$ \\
\hline $\mathrm{F}$ & $\begin{array}{l}3588.099 \\
3586.549\end{array}$ & $(2)$ & III & 27861.96 & -0.02 & $5 d{ }^{3} \mathrm{D}_{2}-7 p^{\prime}{ }^{1} \mathrm{D}_{2}^{\circ}$ & $\mathrm{F}$ & 3119. 202 & $\ldots$ & $3 N$ III & 32050.19 & & \\
\hline $\mathrm{C}$ & 3579.665 & $(20 r)$ & $\begin{array}{l}10 \\
80 n\end{array}$ & $\begin{array}{l}27874.031 \\
27927.633\end{array}$ & $\begin{array}{l}0.00 \\
0.000\end{array}$ & 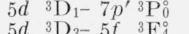 & $\mathrm{F}$ & 3117.638 & & {$[3 n]$ III } & 32066.27 & 0.00 & $5 d{ }^{3} \mathrm{D}_{1}-11 \mathrm{f} \quad{ }^{3} \mathrm{~F}_{2}^{\circ}$ \\
\hline $\mathrm{F}$ & 3577.615 & $\ldots$ & $30 \quad$ III & 27943.60 & -0.09 & $\begin{array}{l}5 d{ }^{3} \mathrm{D}_{3}-5 f{ }^{3} \mathrm{~F}_{4}^{4} \\
5 d \\
3 d \mathrm{D}_{3}-7 p^{\prime}{ }^{3} \mathrm{D}_{3}^{\circ}\end{array}$ & I & $\begin{array}{l}3117.34 \\
3109.63\end{array}$ & - & & $\begin{array}{l}32069.33 \\
32148.84\end{array}$ & 0 & $3 D_{2}-13 f \quad 3 F_{0}^{\circ}$ \\
\hline $\mathrm{F}$ & 3576.036 & $\ldots-\ldots$ & 10 III & 27955.94 & -0.09 & $5 d{ }^{3} \mathrm{D}_{1}-7 p^{\prime}{ }^{3} \mathrm{P}_{1}^{3}$ & $\mathrm{~F}$ & 3108.21 & & $10 n$ & $\begin{array}{l}32148.84 \\
32163.53\end{array}$ & 0.0 & $5 a-D_{2}-131 \quad \mathrm{~F}_{3}$ \\
\hline 1 & 3571.8 & $1 h$ & & 27989. 10 & & & I & 3103.1 & & & 32216.49 & -0.09 & $5 d{ }^{3} \mathrm{D}_{1}-12 f{ }^{3} \mathrm{~F}_{2}^{\circ}$ \\
\hline $\mathrm{F}$ & 3566.660 & $\ldots$ & 10 & 28029.43 & +0.10 & $5 d{ }^{3} \mathrm{D}_{1}-7 p^{\prime}{ }^{3} \mathrm{D}_{2}^{\circ}$ & $\mathrm{C}$ & 3071.583 & $(20)$ & $100 \mathrm{R}$ III & 32547.076 & 0.000 & $6 s^{2}{ }^{1} \mathrm{~S}_{0}-7 p{ }^{1} \mathrm{P}_{1}^{2}$ \\
\hline $\mathrm{F}$ & 3561.942 & $\ldots \ldots$ & III & 28066.56 & -0.10 & $5 d{ }^{3} \mathrm{D}_{2}-5 f,{ }^{1} \mathrm{~F}_{3}^{\circ}$ & $\mathrm{C}$ & 2785. 276 & (2) & 15 IV & 35892.518 & 0.000 & $6 s^{2}{ }^{1} \mathbf{S}_{0}-8 p{ }^{1} \mathrm{P}_{1}^{0}$ \\
\hline I & 3552.3 & $1 h$ & & 28142.74 & & $5 d{ }^{3} \mathrm{D}_{2}-7 p^{\prime}{ }^{3} \mathrm{P}_{2}^{0}$ & $\stackrel{\mathrm{H}}{\mathrm{C}}$ & $\begin{array}{l}2739.243 \\
2702.632\end{array}$ & $\begin{array}{l}4 \\
(2)\end{array}$ & $\begin{array}{l}\text { IV } \\
\text { IV }\end{array}$ & $\begin{array}{l}36495.64 \\
36990.016\end{array}$ & $\begin{array}{r}-0.13 \\
0.000\end{array}$ & $\begin{array}{l}6 s^{2}{ }^{1} \mathrm{~S}_{0}-7 p^{\prime}{ }^{3} \mathrm{D}_{i}^{\circ} \\
6 s^{2}{ }^{1} \mathrm{~S}_{0}-7 p^{\prime}{ }^{3} \mathrm{P}_{i}^{\circ}\end{array}$ \\
\hline $\mathrm{C}$ & 3547.680 & (3) & $20 n$ III & 28179.415 & +0.008 & $5 d \quad{ }^{3} \mathrm{D}_{2}-5 f \quad{ }^{3} \mathrm{~F}_{2}^{\circ}$ & I & 2646.50 & $1 h$ & & 37774.50 & 0.00 & $6 s^{2}{ }^{1} \mathrm{~S}_{0}-9 p{ }^{1} \mathrm{P}_{\mathrm{i}}^{1}$ \\
\hline C & $\begin{array}{l}3544.655 \\
3540.72\end{array}$ & $\begin{array}{c}(20 r) \\
1\end{array}$ & $80 n$ III & $\begin{array}{l}28203.462 \\
28234.77\end{array}$ & +0.013 & $5 d \quad{ }^{3} \mathrm{D}_{2}-5 f \quad{ }^{3} \mathrm{~F}_{3}^{\circ}$ & $\begin{array}{l}\mathrm{C} \\
\mathrm{I}\end{array}$ & $\begin{array}{l}2596.637 \\
2592.30\end{array}$ & (1) & $8 n$ IV & $\begin{array}{l}38499.852 \\
38564.25\end{array}$ & 0.000 & $6 s^{2}{ }^{1} \mathrm{~S}_{0}-7 p^{\prime}{ }^{1} \mathrm{P}_{1}^{\circ}$ \\
\hline $\mathrm{F}$ & 3531.345 & & $30 N$ III & 28309.73 & 0.00 & $5 d{ }^{1} \mathrm{D}_{2}-7 f \quad \mathrm{~F}_{3}$ & I & 2543. 2 & - & & 39308.74 & 0.00 & $6 s^{2}{ }^{1} \mathrm{~S}_{0}-10 p \quad{ }^{1} \mathrm{P}_{1}^{\circ}$ \\
\hline $\mathrm{C}$ & 3529.480 & (3) & 15 III & 28324. 720 & 0.000 & $5 d \quad{ }^{3} \mathrm{D}_{2}-7 p^{\prime}{ }^{3} \mathrm{D}_{3}^{\circ}$ & $\mathrm{I}$ & 2500.2 & - & & 39984.75 & 0.00 & $6 s^{2}{ }^{1} \mathrm{~S}_{0}-11 p{ }^{1} \mathrm{P}_{1}^{0}$ \\
\hline $\mathrm{C}$ & 3524.973 & $(20 r)$ & $80 n$ III & 28360.935 & -0.005 & $5 d \quad 3 \mathrm{D}_{1}-5 f \quad{ }^{3} \mathrm{~F}_{2}^{\circ}$ & I & 2473.20 & $0 h$ & & 40421.23 & 0.00 & $6 s^{2}{ }^{1} \mathrm{~S}_{0}-12 p{ }^{1} \mathrm{P}_{1}^{0}$ \\
\hline $\begin{array}{l}\mathrm{K} \\
\mathrm{C}\end{array}$ & & $\begin{array}{c}0 n \\
(50)\end{array}$ & $200 P$ & 28369.38 & -0.55 & $5 d{ }^{1} \mathrm{D}_{2}-39765^{\circ} ?$ & $\mathrm{H}$ & 2373. 109 & 3 & & 42125.95 & & \\
\hline $\mathrm{F}$ & $\begin{array}{l}3501.107 \\
3463.741\end{array}$ & $(50)$ & $200 R \quad 11$ & 28554.257 & 0.000 & $6 s^{2}{ }^{1} \mathrm{~S}_{0}-6 p^{\prime}{ }^{1} \mathrm{P}_{i}$ & $\mathrm{H}$ & 2216.577 & 2 & & 45100.56 & & \\
\hline $\mathrm{F}$ & 3427.85 & (n) & $\begin{array}{cc}|40| & \text { III } \\
3 n & \text { III }\end{array}$ & $\begin{array}{l}28862.25 \\
29164.44\end{array}$ & $\begin{array}{c}0.00 \\
-\end{array}$ & $5 d{ }^{1}{ }^{1} \mathrm{D}_{2}-8 f{ }^{1}{ }^{1} \mathrm{~F}_{3}^{0}$ & $\mathrm{H}$ & 2214. 639 & 2 & & 45140.02 & & \\
\hline
\end{tabular}

\section{REFERENCES}

A, H. M. Randall, Astrophys. J. 42, 195 (1915).

B, W. F. Meggers, B. S. J. Research 10, 680 (1933) RP 558; and unpublished material (Jan. 1935).

C, F. Sullivan and K. Burns, Science Studies, St. Bonaventure Coll. 9, No. 3, 7 (1941)

D, E. Lorenser, see H. Kayser und H. Konen, Handbuch der Spectroscopie $\boldsymbol{7}$, 79 (1934). F, A. S. King, Contr. Mt. Wilson Solar Obs. No. 150; Astrophys. J. 48, 13 (1918);
G, F. Exner und E. Haschek, see H. Kayser und H. Konen, Handbuch der H, K. Schmitz, see H. Kayser und H. Konen, Handbuch der Spectroseopie $\boldsymbol{\gamma}$,

79 (1934).

J, see A. Fowler, Report on Series in Line Spectra, p. 133 (Fleetway Press, London, 1922).

K, see H. N. Russell and F. A. Saunders, Astrophys. J. 61, 38 (1925) 
TABLE 3. Intensities of Ba I combinations

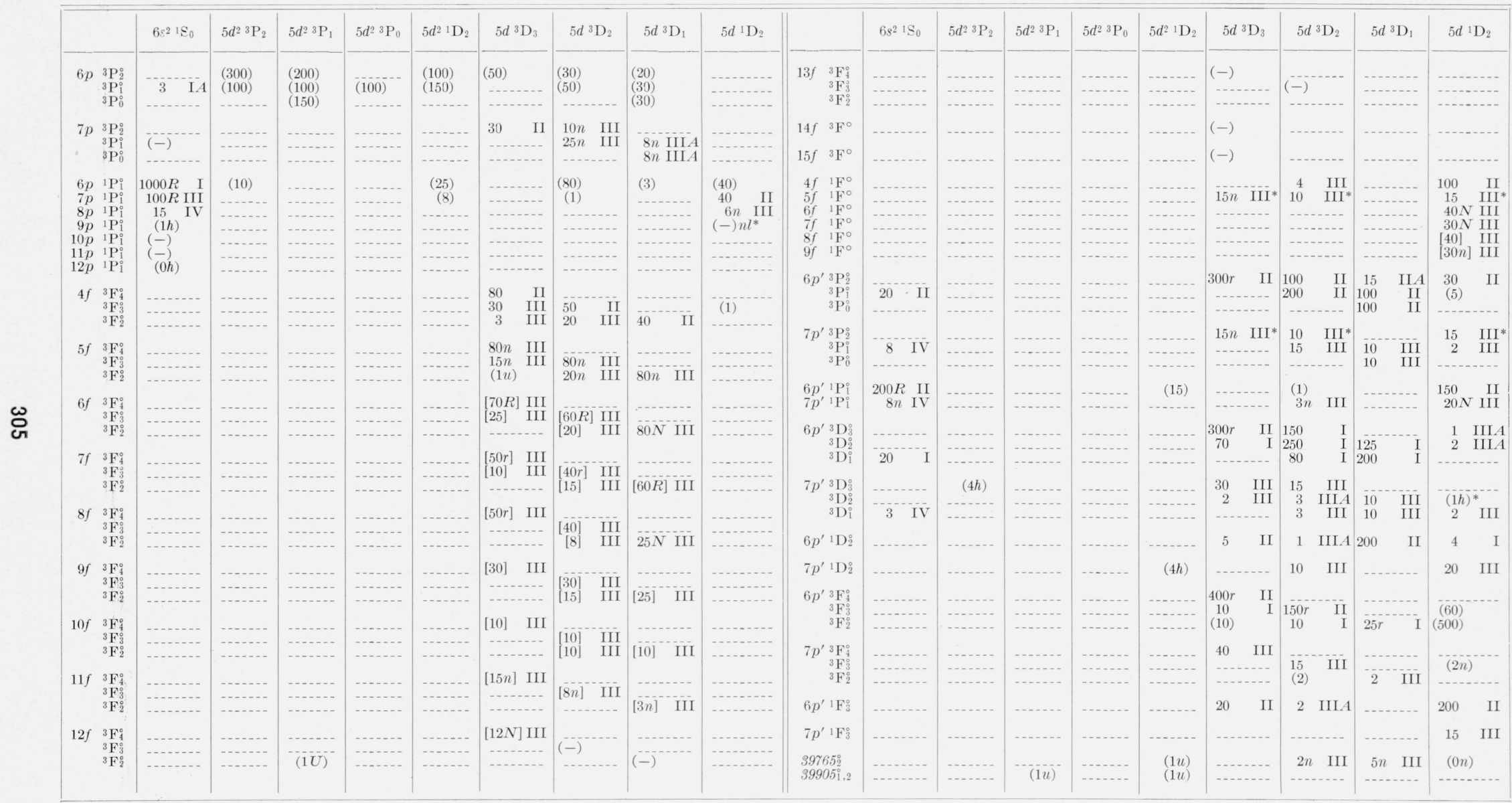

* Blend. 
TABLE 3. Intensities of Ba I combinations-Continued

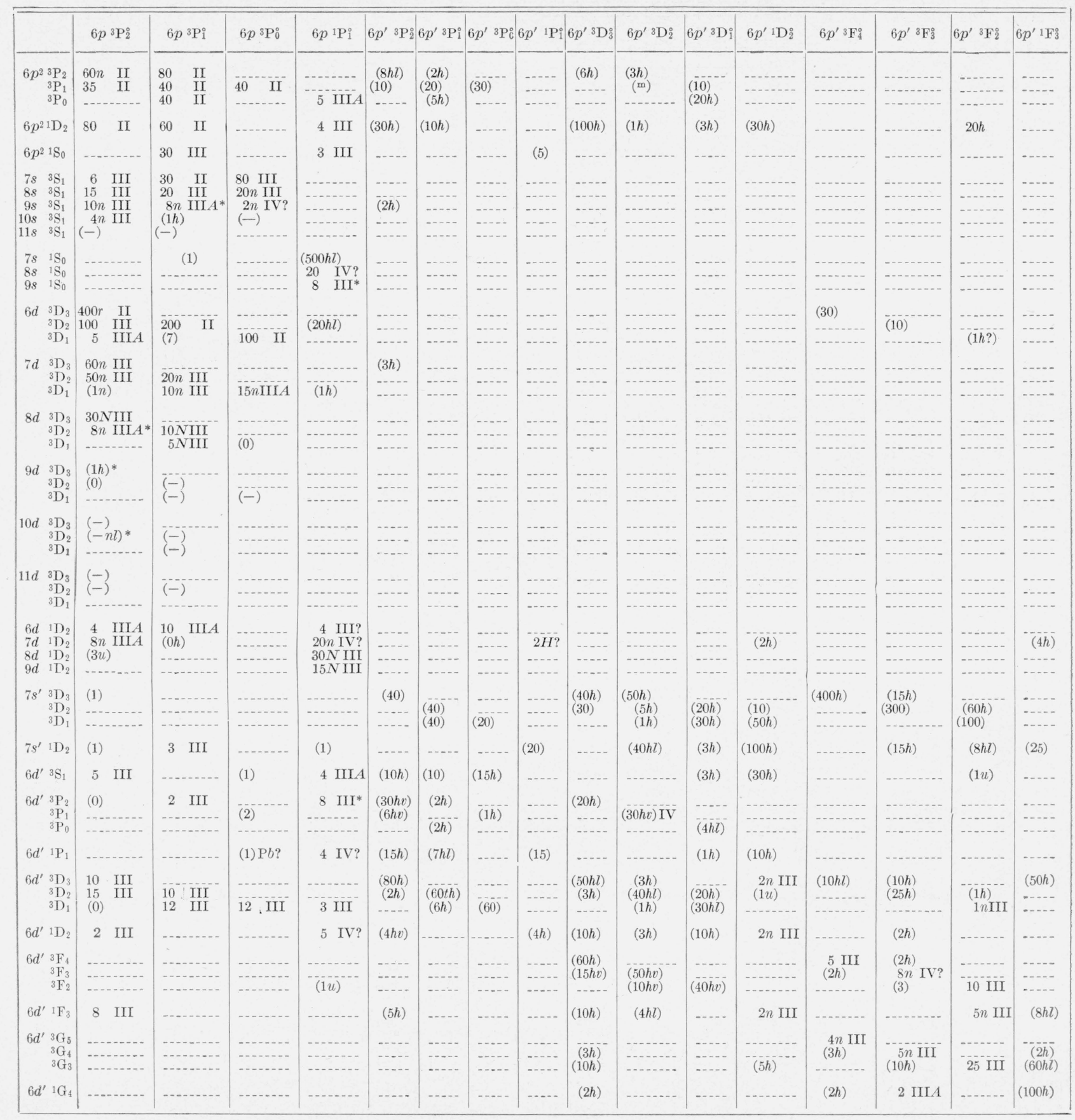

*Blend.

mMasked.

Washington, February 18, 1955. 